\title{
Matryoshka-type gastro-resistant microparticles for the oral treatment of Mycobacterium tuberculosis
}

\author{
Andreu, Vanesa; Larrea, Ane; Rodriguez-Fernandez, Pablo; Alfaro, Salvador; Gracia, Begoña; \\ Lucía, Ainhoa; Usón, Laura; Gomez, Andromeda-Celeste; Mendoza, Gracia; Lacoma, Alicia; \\ Dominguez, Jose; Prat, Cristina; Sebastian, Victor; Ainsa, José Antonio; Arruebo, Manuel
}

Published Online:8 Feb 2019. https://doi.org/10.2217/nnm-2018-0258

\begin{abstract}
Aim: Production of Matryoshka-type gastroresistant microparticles containing antibiotic-loaded PLGA nanoparticles against Mycobacterium tuberculosis. Materials \&methods: The emulsification and evaporation methods were followed for the synthesis of PLGA nanoparticles and methacrylic acid-ethyl acrylate-based coatings to protect rifampicin from degradation under simulated gastric conditions. Results \& Conclusion:The inner antibiotic-loaded nanoparticles here reported can be released under simulated intestinal conditions whereas their coating protects them from degradation under simulated gastric conditions. The encapsulation does not hinder the antituberculosis action of the encapsulated antibiotic rifampicin. A sustained antibiotic release could be obtained when using the drug-loadedencapsulated nanoparticles.Compare to the administration of the free drug, a more effective elimination of M. tuberculosis was observed when applying the nanoparticles against infected macrophages. The antibiotic-loaded PLGA nanoparticles were also able to cross an in vitro model of intestinal barrier.
\end{abstract}

\section{Graphical abstract}

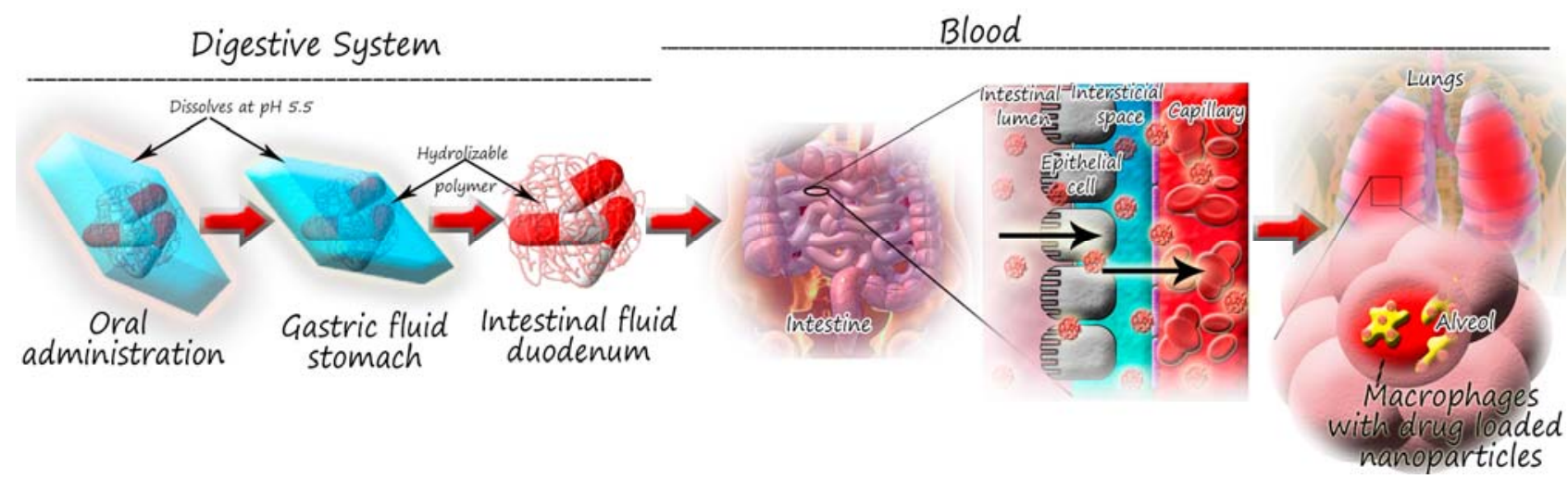

Graphical Abstract Description- Matryoshka-type gastroresistant microparticles containing antibiotic-loaded PLGA nanoparticles against Mycobacterium tuberculosis were produced to protect the antibiotic from degradation under simulated gastric conditions. The antibiotic-loaded PLGA nanoparticles were able to cross an in vitro model of intestinal barrier, being more effective in the elimination of $M$. tuberculosis when applied against infected macrophages compared to the use of the free drug.

Keywords:Tuberculosis; PLGA; microparticles; rifampicin; Eudragit; enteric coating; mycobacterium tuberculosis; oral delivery; gastroresistant; TEER

\section{Main body of text:}

Mycobacterium tuberculosis is a well-known example of a highly spread pathogen causing tuberculosis (TB), a disease that greatly affects public health in most developing countries. $M$. tuberculosis is localized in the early endosomes of infected macrophages[1]. According to the last WHO report[2], despite the moderate decline (1.5\%) in the global incidence of TB observed during the last 15 years, being in 2015 a 18\% lower than in 2000, TB still constitutes a major cause of morbidity and mortality worldwide. In 2016, there were 10.4 million new cases and 1.7 million people died from the disease worldwide ranking alongside HIV as a leading cause of death. In the treatment of drug susceptible TB, in order to act on different bacterial subpopulations (extracellular and intracellular), current treatment is extended over long periods of time (6-9 months) with a combination of at least four of the five first-line drugs against the pathogen in order 
to prevent both relapse and development of antibiotic resistance. Rifampicin, isoniazid, pyrazinamide and ethambutol are given daily usually during the first two months of treatment, leaving just rifampicin and isoniazid for the remaining period. Except for pyrazinamide, the other commonly used antibiotics require bacterial replication for their action[3].

Being at least partially an intracellular pathogen, any treatment against TB should be selectively directed towards the intracellular reservoir of the bacterial load. The introduction of targeted drug delivery systems into the clinical practice for treating intracellular pathogens would allow applying drugs effectively, increasing patient compliance, extending the product life cycle, providing product differentiation and reducing healthcare costs. Over the last decades, significant progress has been made in the development of nanotechnological approaches to diagnose, screen, treat, and prevent disease, hence improving human health. As a consequence of this extensive research 43 nanomedicines have been approved for the clinical practice by the EMA and 71 by the US FDA mainly as drugs and medical devices and other 128 are currently undergoing clinical trials [4]. Their application is principally directed towards cancer treatment and antimicrobial therapy using drug delivery systems. In those systems the nanoparticle (NP) acts as a drug depot either as suspensions or as coatings on medical devices. General applications of nanomaterials in the field of infectious diseases include their use as adjuvants or as antimicrobial and vaccine carriers, to achieve a sustained or controlled delivery depending on the need. In addition, several metallic nanomaterials (i.e., silver, copper, etc.) are antimicrobial by themselves releasing, after oxidation, metallic ions that show multiple mechanisms of biocidal action and consequently avoiding antimicrobial resistance. When multiple mechanisms of action take place against a pathogenic microorganism simultaneous gene mutations are difficult to occur and consequently the development of resistance is reduced. In addition, nanoparticles can be used to achieve a targeted and selective delivery of the corresponding antimicrobial drug. Targeted strategies can be used by coupling the antimicrobial NP to a specific recognition moiety (i.e., antibody, peptide, oligonucleotides, etc.), or by electrostatic binding to the bacterial wall [5]or even the antimicrobial can be released just in response to a biomolecule secreted under the presence of bacteria [6,7]. Passive strategies take advantage of the nanoparticle-immune system recognition. Thus, macrophages detect, internalize and destroy not only pathogens and apoptotic cells, but also NPs due to the large number of non-specific surface receptors that those cells have[3]. NPs are internalized within those cells using the phagocytic route when proteins of the blood plasma (i.e., opsonins) are adsorbed on the NP surfaces. Also, other internalization non-phagocytic routes (clathrin or caveolin-mediated endocytosis, macropinocytosis, etc.) can internalize different NPs within intracellular vesicles. Therefore, NPs can accumulate their antimicrobial cargos into intracellular compartments of infected cells. In vitro and in vivo experiments showed enhanced antimicrobial activity of antibiotic containing NPs compared to the administration of the free drug; however, a specific colocalization of the NP and the pathogen in the same intracellular compartment is difficult to achieve. In this regard, Kalluru et al. [8] demonstrated that even though rifampicin loaded poly lactic-co-glycolic acid (PLGA) nanoparticles were able clear macrophages infected with Mycobacterium bovis (BCG) more efficiently compared to the administration of the [9] free drug, the drug-containing NPs remained inside phagolysosomes that were separated from the BCG-containing phagosomes. The authors postulated that the loaded hydrophobic drugs easily crossed by diffusion both, the phagolysosome membrane enclosing the NP (phagosome to cytoplasm) and the membrane of the phagosome enclosing the BCG. Therefore, a perfect subcellular co-localization inside the same vesicle is not necessary to show enhanced efficacy. In addition, the great benefit of using nanomaterials is that by selecting a specific size [10] or by using a simple surface modification, the same NP can be internalized following different routes [11].

Oral administration of drugs is preferable over any other route of administration because it is not invasive and shows long term compliance and increased patient acceptance, allowing easily home therapy [12]. The main drawback of this route is the potential degradation of the active principle in contact with the acidic and enzymatic environment of the gastrointestinal tract and during the pre-systemic metabolism. Capsules have been designed to protect the drug from the degradation in the gastric fluid releasing its cargo in the intestine in a pH-responsive manner. For instance, Patel et al. [13] described the synthesis and sustained rifampicin release from $\mathrm{pH}$-dependent chitosan-based nanoparticles intended for oral delivery. Also, $\mathrm{pH}$-independent sustained release formulations have been designed to increase the intestinal adsorption using mucoadhesive formulations and absorption enhancers.

The activity of rifampicin is based on the inhibition of the RNA synthesis, by means of van der Waals forces, of the hydrophobic side chains near the naftol ring of the rifampicin with the bacterial DNA-dependent RNA polymerase[14]. Rifampicin is degraded ( 26\%) under gastric conditions, which can be responsible of its reduced bioavailability in vivo, but its nanoparticulated formulation can reduce the degradation level to a $9 \%$ [15]. NPs have also been used to protect and transport active principles across the intestinal epithelium to the bloodstream by targeting them with specific proteins able to cross polarized epithelial barriers [16] or by using appropriated sizes surface and charges opening the tight junctions between the epithelial cells [17].

In this work, we describe a drug delivery system potentially able to reach infected alveolar macrophages using biodegradable and approved antibiotic-loaded carriers. The system is based on nanoparticulated formulations of PLGA (Resomer ${ }^{\circledR}$ RG504) loaded with rifampicin targeting M. tuberculosis. Those drug-loaded NPs are, in a second step, encapsulated inside (as Matryoshka dolls) microparticles (MPs) based on methacrylic acid-ethyl acrylate copolymer (1:1, 
Eudragit $\left.{ }^{\circledR} \mathrm{L} 100-55\right)$ to cause the drug loaded NPs to first pass through the stomach without being dissolved (thanks to the shell made of Eudragit ${ }^{\circledast}$ L 100-55) to then dissolve in the intestinal fluid releasing the inner NPs made of PLGA. PLGA was selected as a polymer due to its well-known properties: 1) predictable biodegradability and biocompatibility, 2) US Food and Drug Administration and European Medicine Agency approvals for its application as building material in drug delivery systems, 3) well-described formulations and methods of production adapted to the encapsulation of various types of hydrophilic or hydrophobic active pharmaceutical ingredients, 4) drug protection from biochemical degradation, 5) possibility to target its based nanoparticles (NPs) to specific tissues or cells, and 6) the possibility of achieving a sustained release. Nanoparticulated formulations of PLGA can be produced by nanoprecipitation and emulsification with solvent evaporation, being the procedures that have been actively used during the last three decades to produce drug-loaded PLGA nanoparticles. Emulsification with solvent evaporation was selected against nanoprecipitation because by nanoprecipitation it was difficult to couple the rifampicin and the PLGA precipitation. On the contrary, by emulsification, rifampicin was selectively loaded inside the PLGA emulsion and afterwards it was entrapped during the precipitation of the polymer once the solvent was evaporated. Some other production techniques of drug loaded nanoparticulated formulations, such as emulsion crosslinking or post drug loading, were not considered due to the following concerns:

1) PLGA is a polymer with rare possibilities to be crosslinked, unless it is functionalized. In this work, Resomer ${ }^{\circledR}$ RG504 was used and no further functionalization was feasible because it is an ester terminated polymer.2) Cross-linking of polymers is conducted via a controlled chemical reaction. It implies that not only the polymer but also the drug could react with the cross linker agent. If this would be the case, the drug could not have any therapeutic effect and even could be more cytotoxic that the unmodified drug.3)Post-drug loading implies that the drug molecules should permeate through the polymeric shell of the nanoparticle. Then, it would be required a large drug concentration gradient to enable a sufficient drug loading. Considering that the drug release in PLGA is powered by the PLGA hydrolysis, the PLGA post-drug loading should require a larger processing time than the one selected by solvent evaporation, as well as a more expensive sorting procedure to separate the unloaded drug from the polymer and the surfactant molecules used.

\section{Materials \& methods}

\section{Nano and microparticle synthesis}

The polymer, Eudragit ${ }^{\circledR}$ L 100-55 is an anionic methacrylic acid-ethyl acrylate copolymer (1:1) containing free carboxylic groups which are ionized in an aqueous media at $\mathrm{pH} \geq 5.5$ making it resistant to the acid environment of the gastric fluid, but slightly soluble at intestinal (duodenum) pH. Resomer ${ }^{\circledR}$ RG504 is an ester terminated poly(D,L-lactide-co-glycolide) 50:50 (Mw 38000$54000 \mathrm{Da}$ ) widely used in controlled release systems. Both polymers were gifted by Evonik Industries AG (Darmstadt, Germany) and used as received. Drugs including rifampicin and dexamethasone (as internal standard); surfactants including sodium cholate hydrate and Pluronic ${ }^{\circledR}$ F-68; and solvents including ethyl acetate, ethanol absolute, acetonitrile and methanol (HPLC grade) were supplied by Sigma Aldrich (St. Louis (MO), USA).

PLGA-rifampicin NPs were prepared by the oil-in-water (O/W) emulsion solvent evaporation method. In this method, $1 \%(\mathrm{w} / \mathrm{v})$ of PLGA polymer (50:50), $0.2 \%(\mathrm{w} / \mathrm{v})$ of rifampicin and $3 \%(\mathrm{w} / \mathrm{v})$ of Pluronic F68, used as surfactant, were dissolved in $5 \mathrm{~mL}$ of ethyl acetate. The organic phase was then emulsified with $10 \mathrm{~mL}$ of Milli $Q$ water used as aqueous phase. This mixture was sonicated in an ice bath for 25 seconds with a sonicator (Digital Sonifier 450, Branson, USA) using a probe of 0.13 inches in diameter and $40 \%$ of amplitude. After the formation of a stable emulsion, the organic solvent was evaporated under continuous stirring (600 rpm) for 3 $\mathrm{h}$ to allow polymer precipitation.

Microcapsules based on Eudragit were prepared by following the double emulsion solvent evaporation method (w/o/w) using antibiotic loaded PLGA NPs in the inner water phase. Briefly, 2\% (w/v) of Eudragit L100-55 was dissolved in $2.5 \mathrm{~mL}$ of ethanol:ethyl acetate (1:4) (organic phase) and emulsified with $1 \mathrm{~mL}$ of the antibiotic-loaded PLGA suspension ( $25 \mathrm{mg} / \mathrm{mL}$ ) (inner aqueous phase) by ultrasonication (Digital Sonifier 450 , Branson, USA) at a $40 \%$ of amplitude for 30 seconds in an ice bath. The formed w/o emulsion was then emulsified with $2 \mathrm{~mL}$ of a $1 \%(\mathrm{w} / \mathrm{v})$ sodium cholate solution at $40 \%$ amplitude for 35 seconds to obtain the $\mathrm{w} / \mathrm{o} / \mathrm{w}$ emulsion. Ten millilitres of $0.3 \%(\mathrm{w} / \mathrm{v})$ sodium cholate solution were also added before stirring to promote the stability of the final emulsion.

\section{Antimicrobial evaluation}

For determining the antituberculosis activity of the samples, mycobacterial strains were grown at $37{ }^{\circ} \mathrm{C}$ in Middlebrook $7 \mathrm{H} 9$ broth supplemented with $10 \% \mathrm{ADC}$ and $0.05 \%$ Tween 80 until reaching an $\mathrm{OD}_{600}$ of 0.8 . Then, bacterial cultures were diluted in Middlebrook $7 \mathrm{H} 9$ broth supplemented with $\mathrm{ADC}$ and $0.5 \%$ glycerol in order to obtain a bacterial suspension of $10^{5} \mathrm{CFU} / \mathrm{mL}$. Aliquots of $100 \mu \mathrm{L}$ were transferred to each well of a 96-wells plate that contained $100 \mu \mathrm{L}$ of samples prepared from 2-fold serial dilutions in the same culture medium. The inoculated plates were incubated for 6 days at $37^{\circ} \mathrm{C}$ and for an additional 2 days after the addition of the redox indicator $(30 \mu \mathrm{L}$ of a resazurin solution at $0.1 \mathrm{mg} / \mathrm{mL}$ ). A change from blue to pink indicates reduction of 
resazurin and therefore bacterial growth. Thus, the MIC (minimum inhibitory concentration) was defined as the lowest concentration of compound that prevented this colour change.

For estimating the release of rifampicin from NPs, $0.806 \mathrm{mg} / \mathrm{mL}$ of rifampicin-loaded PLGA NPs were incubated in $400 \mu \mathrm{L}$ of culture media (Middlebrook $7 \mathrm{H} 9$ broth supplemented with $\mathrm{ADC}$ and $0.5 \%$ glycerol) adjusted at $\mathrm{pH}=7.0$ or $\mathrm{pH}=4.5$, at $37^{\circ} \mathrm{C}$. Samples were taken after $3,7,14$, and 25 days, centrifuged for pelleting (10 min at $4500 \mathrm{rpm}$ ) and the NPs and the supernatants transferred to a new tube and kept frozen until use. Once all samples were ready, the supernatants were evaluated for the presence of rifampicin by using the protocol described above. As a negative and sterility control, $0.554 \mathrm{mg} / \mathrm{mL}$ of empty PLGA NPs were processed in the same way as rifampicin-loaded NPs were. In order to consider spontaneous degradation of rifampicin after being released from the NPs, tubes containing an amount of free rifampicin equal to that encapsulated in the PLGA NPs were incubated and processed in the same way. The experiments were performed in triplicate.

\section{Bacterial growth and time-kill kinetics}

The time-kill kinetics of $M$. tuberculosis H37Rv were determined by incubating cultures with NPs and free rifampicin and counting the number of viable bacteria at several time points [18]. Mycobacterial cells were cultured till exponential phase and then diluted to reach $10^{7} \mathrm{CFU} / \mathrm{mL}$ in Middlebrook $7 \mathrm{H} 9$ broth. Four samples of $10 \mathrm{~mL}$ of this bacterial suspension were taken separately and added with: i) rifampicin, to reach an inhibitory concentration of $0.5 \mu \mathrm{g} / \mathrm{mL}$, ii) rifampicin-loaded NPs, which contained rifampicin for final inhibitory concentrations of $0.06-0.07 \mu \mathrm{g} / \mathrm{mL}$, and 4.9-6.0 $\mu \mathrm{g} / \mathrm{mL}$ of PLGA (which are below inhibitory concentrations of PLGA), iii) empty PLGA NPs to reach the same final concentration of $4.9-6.0 \mu \mathrm{g} / \mathrm{mL}$; the fourth sample did not receive any compound and was taken as control. All four samples were incubated at $37^{\circ} \mathrm{C}$. On days $3,7,14$, and 21 , from each sample, an aliquot of $100 \mu \mathrm{L}$ was taken for diluting and plating on Middlebrook 7H10 agar plates which were incubated further at $37^{\circ} \mathrm{C}$ until growth was observed in order to count the number of CFUs. The assay was performed in duplicate.

Loaded or empty nanoparticles at sub-inhibitory concentrations of PLGA and rifampicin (in the case of the loaded nanoparticles) were added to cultures of $M$. tuberculosis in order to estimate their impact on bacterial growth. Bacterial cells were cultured till exponential phase; they were diluted to reach $10^{5}-10^{6} \mathrm{CFU} / \mathrm{mL}$ in fresh Middlebrook $7 \mathrm{H} 9$ broth, and separated into three samples of $10 \mathrm{~mL}$. PLGA NPs containing rifampicin equalling one-fourth of its MIC (i.e., $0.004 \mu \mathrm{g} / \mathrm{mL}$; MIC $=0.015 \mu \mathrm{g} / \mathrm{mL}$ ) was added to the first culture; the second was added with the same amount of empty PLGA NPs (to reach $0.32 \mu \mathrm{g} / \mathrm{mL}$ of PLGA, well below its MIC, which is $7.8 \mu \mathrm{g} / \mathrm{mL}$ ); the last sample was incubated without further additions, as control. Immediately, an aliquot was removed for determining initial values of $\mathrm{OD}_{600}$ and $\mathrm{CFU} / \mathrm{mL}$. Cultures were incubated at $37{ }^{\circ} \mathrm{C}$ and new aliquots were taken and analyzed for $\mathrm{OD}_{600}$ and $\mathrm{CFU} / \mathrm{mL}$ after $3,5,7,10,12,14$ and 21 days.

\section{Cell culture}

TC7 clone human epithelial colorectal adenocarcinoma (Caco2) cells, kindly donated by Dr MJ Rodriguez-Yoldi, were grown using Dulbecco's modified Eagle's medium (DMEM w/stable Glutamine; Biowest) in a humidified atmosphere containing $5 \% \mathrm{CO}_{2}$ at $37{ }^{\circ} \mathrm{C}$. The growth medium was supplemented with fetal bovine serum (FBS, $10 \%$ (v/v); Thermo Fisher Scientific) and antibioticantimycotic ( $60 \mu \mathrm{g} / \mathrm{mL}$ penicillin, $100 \mu \mathrm{g} / \mathrm{mL}$ streptomycin and $0.25 \mu \mathrm{g} / \mathrm{mL}$ amphotericin B, Biowest).

MH-S murine alveolar macrophages were maintained in Roswell Park Memorial Institute medium (RPMI) 1640 supplemented with FBS $10 \%$ at $37^{\circ} \mathrm{C}$ in a $5 \% \mathrm{CO}_{2}$ humidified incubator.

\section{Cell viability assay}

The in vitro cytotoxicity of rifampicin, unloaded-PLGA NPs and rifampicin-loaded PLGA NPs was evaluated using the resazurin based Alamar Blue ${ }^{\mathrm{TM}}$ assay (Invitrogen, US) according to the manufacturer's instructions. TC7 Caco2 (density of 8000 cells/well) cells were seeded in a 96-well plate $48 \mathrm{~h}$ prior to incubation with the different formulations and allowed to adhere. Non-loaded-PLGA and rifampicin-loaded PLGA NPs were added to the cells in complete growth medium at a concentration range of $0.0625-1 \mathrm{mg} / \mathrm{mL}$ and, then, cells were maintained in the standard conditions mentioned above for $24 \mathrm{~h}$. Rifampicin was added to the cells at a concentration range of $0.5-8 \mu \mathrm{g} / \mathrm{mL}$, which are equivalent drug concentrations to the ones contained in the rifampicin-loaded PLGA nanoparticles used. After incubation, cells were washed twice with Dulbecco's Phosphate Buffered Saline (DPBS, Biowest) and incubated with complete growth medium containing $10 \%(\mathrm{v} / \mathrm{v})$ Alamar Blue dye for $4 \mathrm{~h}$. Fluorescence of the medium was measured at $37{ }^{\circ} \mathrm{C}$ in a multi-mode Synergy HT Microplate Reader (excitation wavelength $530 \mathrm{~nm}$, emission wavelength $590 \mathrm{~nm}$; Biotek). Cell viability was expressed as a relative percentage to the untreated control cells value. The percentages obtained depict the average of four values.

To evaluate the cytotoxic effect of the PLGA NPs on the MH-S cell line, the EZ4U cell proliferation assay (Biomedica) was used following the manufacturer's instructions. Briefly, MH-S were seeded at a concentration of $1 \times 10^{5}$ cells per well in 96 -well tissue culture plates with clear bottoms (Falcon ${ }^{\circledR}$ ) using RPMI without phenol red. The plates were incubated overnight $\left(37^{\circ} \mathrm{C}\right.$ in $5 \% \mathrm{CO}_{2}$ atmosphere). Non-loaded-PLGA NPs, rifampicin-loaded PLGA NPs or free rifampicin at minimum[19,20]and maximum[21-23]serum 
concentrations ( 4 and $24 \mu \mathrm{g} / \mathrm{mL}$, respectively), were diluted in $200 \mu \mathrm{L}$ of RPMI without phenol red per well and incubated for $24 \mathrm{~h}$. The monolayers were washed once with DPBS and then $200 \mu \mathrm{L}$ of fresh medium without phenol red and $20 \mu \mathrm{L}$ of EZ4U were added. The absorbance was read after $4 \mathrm{~h}$ of incubation, and also at $48 \mathrm{~h}$ and $72 \mathrm{~h}$. A microplate reader (Victor 3, Wallac) was used with a wavelength of $450 \mathrm{~nm}$ with $620 \mathrm{~nm}$ as reference. The results were expressed as a viability percentage using untreated cells as control.

\section{Permeability experiments}

For the permeability experiments, TC7 Caco2 cells were seeded on polycarbonate membrane inserts $\left(0.47 \mathrm{~cm}^{2} ; 0.4 \mu \mathrm{m}\right.$ pore size) into a 24 -well plate at a density of $5 \times 10^{4}$ cells $/ \mathrm{cm}^{2}$. Cells were maintained in complete medium for 28 days, replacing it every other day, to obtain well-differentiated monolayers.

The integrity of the epithelial monolayers was evaluated by measuring the Transepithelial Electrical Resistance (TEER). TEER values were measured every 3-4 days using a Millicell ERS2 Voltohmmeter (Millipore) according to manufacturer's instructions throughout the experiment course. The final TEER values were obtained by multiplying the meter readings by the effective surface area of the filter membrane and were expressed as $\Omega \mathrm{cm}^{2}$. TEER values $\geq 400 \Omega \mathrm{cm}^{2}$ over background were used as a reference point to indicate adequate monolayer integrity in order to carry out permeability assays with the TC7 Caco 2 monolayers.

After verification according to TEER values, the integrity of the monolayer was measured by monitoring the Lucifer Yellow (LY) rejection, a paracellular marker, across the cell monolayer. Prior to the experiment, the inserts were washed twice with calciumand magnesium-free Hank's balanced salt solution (HBSS; Biowest) and equilibrated for 60 min with pre-warmed HBSS containing $10 \mathrm{mM}$ HEPES. Immediately, $300 \mu \mathrm{L}$ of $400 \mu \mathrm{M}$ Lucifer yellow and $600 \mu \mathrm{L}$ HBSS containing $10 \mathrm{mM}$ HEPES were added into the apical and basolateral chambers, respectively. The plate was placed at $37{ }^{\circ} \mathrm{C}$ in a $5 \% \mathrm{CO}_{2}$ humidified incubator for $1 \mathrm{~h}$. After the finalization of permeability experiment, LY fluorescence in apical and basal compartments was measured at 485/535 nm excitation /emission wavelengths using a multi-mode Synergy HT Microplate Reader (Biotek) and the percentage rejection of LY was calculated.

Previous to the beginning of the transport experiments, the culture media in the donor and acceptor compartments were aspirated, cells were rinsed twice with pre-warmed HBSS and, immediately, were incubated with HBSS supplemented with 10 mM HEPES and allowed to equilibrate in the transport medium at $37{ }^{\circ} \mathrm{C}$ for $30 \mathrm{~min}$. To evaluate transepithelial transport of rifampicinloaded PLGA nanoparticles, $300 \mu \mathrm{L}$ of nanoparticles suspensions of different concentrations $(2.5,5$ and $10 \mathrm{mg} / \mathrm{mL})$ in transport buffer were loaded into the apical chamber and $600 \mu \mathrm{L}$ HBSS containing $10 \mathrm{mM}$ HEPES were added into the basolateral chamber. Then, cells were incubated at $37{ }^{\circ} \mathrm{C}$ in a $5 \% \mathrm{CO}_{2}$ humidified incubator for $4 \mathrm{~h}$. Then, $300 \mu \mathrm{L}$ of the basolateral solution were collected and the rifampicin concentration was determined by HPLC following the analytical method described in section 2.2 . Briefly, samples containing rifampicin-loaded PLGA NPs were mixed with $1 \mathrm{~mL}$ of acetonitrile and sonified in an ultrasonic bath for 30 minutes. Thereafter, $100 \mu \mathrm{L}$ of an internal standard (dexamethasone, $300 \mathrm{ppm}$ ) was added and the mixture was shaken in a rotator mixer for 15 minutes at $4{ }^{\circ} \mathrm{C}$. After the addition of $100 \mu \mathrm{L}$ of methanol, the solution was mixed in an ultrasonic bath during 15 minutes. Finally, the mixture was filtered using a $0.22 \mu \mathrm{m}$ PTFE syringe filter and placed in a vial for UV-HPLC analysis.

\section{In vitro infection of MH-S macrophages}

MH-S murine alveolar macrophages were plated at a concentration of $3 \times 10^{5}$ cells per well in 24-well tissue culture plates with clear bottoms (TTP ${ }^{\circledR}$ ) and allowed to adhere overnight. For the infection, mid-log phase $M$. tuberculosis was washed twice with PBS with a $0.05 \%$ Tween, once with Dulbecco's PBS and then allowed to stand for $5 \mathrm{~min}$, before the supernatant was collected. The bacteria were then diluted in RPMI and added to the MH-S cells at a concentration of $\sim 3 \times 10^{4} \mathrm{CFU} /$ well. After $3 \mathrm{~h}$ of infection at 37 ${ }^{\circ} \mathrm{C}$ in $5 \% \mathrm{CO}_{2}$, macrophages were treated with $200 \mu \mathrm{g} / \mathrm{mL}$ amikacin for $1 \mathrm{~h}$ and washed three times with DPBS to eliminate any extracellular bacteria. Lastly, $1 \mathrm{~mL}$ of complete RPMI-1640 was added to each well and incubated for $72 \mathrm{~h}$ at $37{ }^{\circ} \mathrm{C}$ in a $5 \% \mathrm{CO}_{2}$ humidified incubator, in order to allow bacteria to grow inside the macrophages. After such incubation (day 0 ), fresh medium containing unloaded-PLGA NPs, rifampicin-loaded PLGA NPs or free rifampicin was added at 4 and $24 \mu \mathrm{g} / \mathrm{mL}$ (minimum[19,20]and maximum[21-23] serum concentrations). The quantity of rifampicin-loaded PLGA NPs added was calculated according to the rifampicin concentration inside the NPs. The quantity of empty PLGA NPs added was proportional to the quantity of loaded PLGA NPs. Intracellular survival and growth were assessed by lysing the monolayers (day 0, 2 and 4) by the addition of water followed by a $30 \mathrm{~min}$ incubation at $37^{\circ} \mathrm{C}$ and enumeration of bacteria by serial dilutions in PBS-Tween plating onto Middlebrook $7 \mathrm{H} 10$ solid medium. Colonies were counted after $3-4$ weeks incubation at $37^{\circ} \mathrm{C}$ and the average $\mathrm{CFU} / \mathrm{mL}$ determined.

\section{Results}

\section{Physico-chemical characterization of the micro- and nanoparticles produced}

Figure 1a shows schematically the experimental protocol used in the synthesis of the antibiotic-loaded PLGA NPs here reported. Single emulsion was used to entrap and preserve the hydrophobic structure and activity of rifampicin. The colloidal hydrodynamic size measured in water was $145.2 \pm 31.2 \mathrm{~nm}$. The measured SEM histograms revealed a particle 
size distribution centred at $149.1 \pm 35.6 \mathrm{~nm}$ (Figures $1 \mathrm{~b}-\mathrm{c}$ ) for the rifampicin-loaded PLGA NPs. As can be seen in Figure S1 (supporting information section), the NP monodispersity was maintained in different independent batches, indicative of the robustness of the synthesis method.

HPLC analysis indicated that the EE and DL achieved for the rifampicin-loaded PLGA NPs were $5.4 \pm 0.55$ and $1.08 \pm$ $0.11 \mathrm{w} / \mathrm{w} \%$, respectively. This DL is similar to those previously reported in the literature using PLGA [24] although higher values have been previously reported with other carriers [13].To check the potential interaction between the antibiotic studied and the encapsulating PLGA NPS, FTIR analysis was performed by comparing the signals of the antibioticencapsulated nanoparticles with a mixture of the corresponding free antibiotic and unloaded PLGA NPs as control under the same proportional concentrations. Figure S2 (supporting information section) shows that the vibrations characteristic of the chemical bonds of the encapsulated antibiotic were similar to the ones retrieved from the simple physical mixture composed of the corresponding antibiotic and empty PLGA NPs. This is indicative that no new chemical bonds between the antibiotic and the PLGA encapsulating matrix were formed. This is a positive result as we preserved during the encapsulating process the chemical structure of the antibiotic in order to maintain its antituberculosis activity.

a)

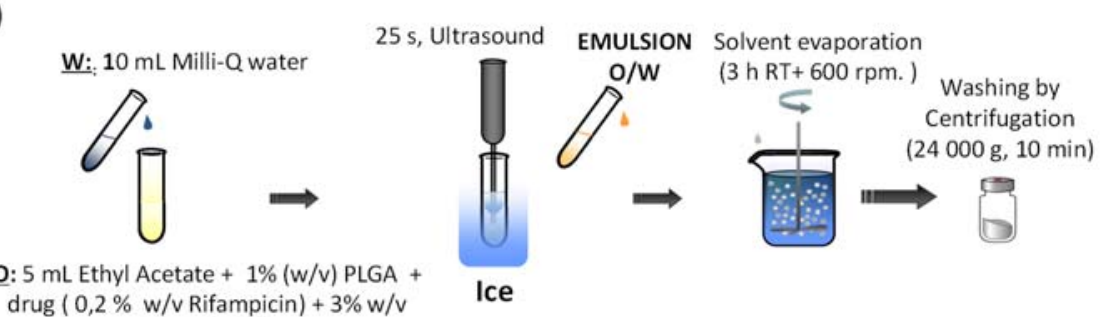
Pluronic F68
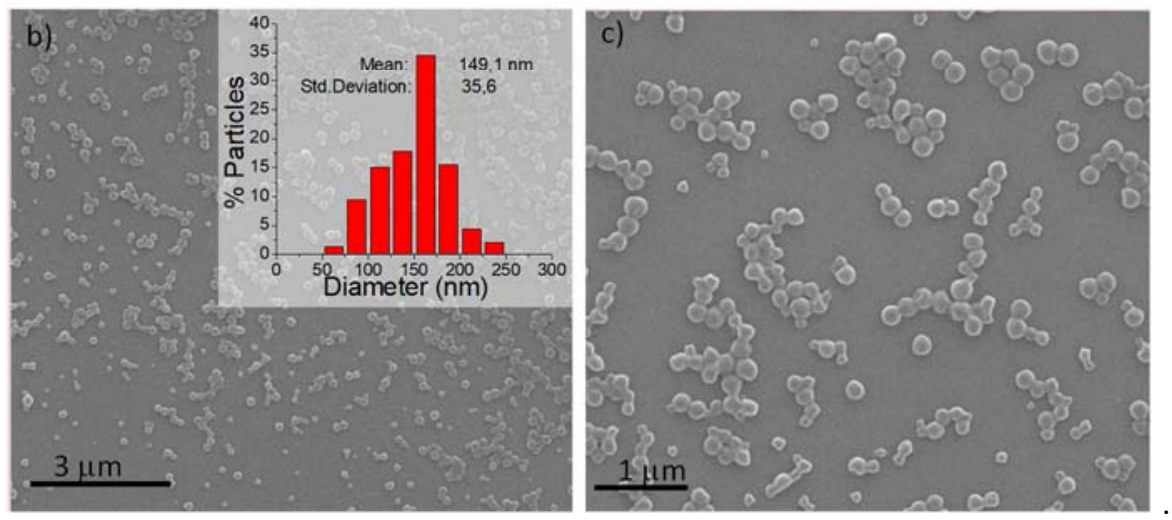

Figure 1. a) Synthesis procedure for the preparation of drug loaded PLGA NPs by single o/w emulsification and evaporation process. b)-c) SEM images of rifampicin loaded PLGA NPs and their corresponding size distribution.

The schematic representation of the Matryoshka-type microparticles reported is shown in Figure 2a where the potential application of the developed nanoparticles for an efficient and controlled intracellular delivery of antibiotics against intracellular alveolar infected macrophages after oral administration is represented. In addition, the schematic synthesis based on the double w/o/w emulsification and evaporation method for the production of the gastroresistant microcapsules containing the inner drug loaded-PLGA NPs is represented in Figure 3a. The SEM morphological analysis of the gastroresistant microcapsules made of Eudragit ${ }^{\circledR}$ L 100-55 with or without inner antibiotic-loaded PLGA nanoparticles (Figures $2 \mathrm{~b}$ and $2 \mathrm{c}$, respectively) revealed a bipyramidal structure of micrometric size $(6.1 \pm 1.9 \mu \mathrm{m})$. We observed that depending on the temperature and consequently on the viscosity of the precursor polymeric solution different morphologies were obtained; probably, thermal fluctuations on the formation of those polymeric shells might be responsible for this polyhedric morphology.

a)

Digestive System Blood

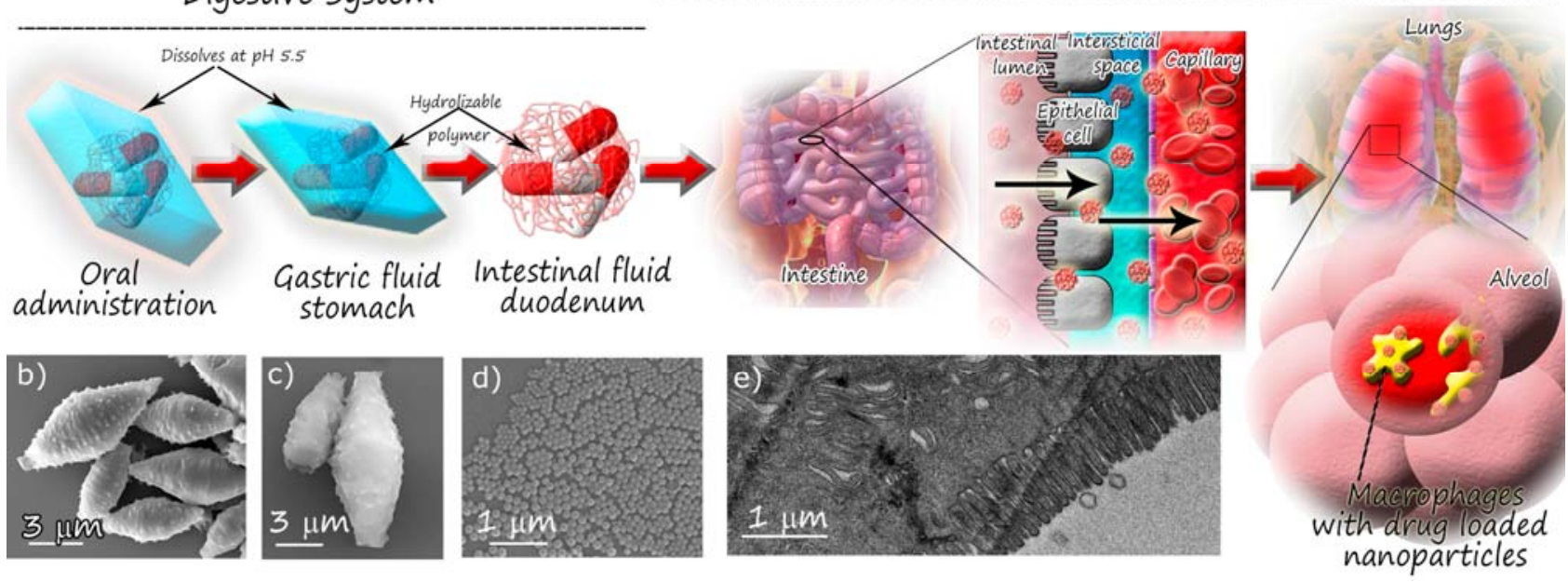


Figure 2. a) Scheme of the envisaged fate of the pH-responsive Matryoshka-like micro- and nanoparticles for an efficient and controlled intracellular delivery of antibiotics against intracellular alveolar infected macrophages. b) SEM of Eudragit microcapsules containing drug-loaded PLGA nanoparticles. c) SEM of Eudragit microcapsules after $2 \mathrm{~h}$ in $\mathrm{HCl} 0.1 \mathrm{~N}$ at 37 ㄷ (simulated gastric conditions). d) SEM of the rifampicin-loaded PLGA nanoparticles loaded inside the external microcapsules. e) TEM image of morphology of TC7 Caco2 epithelial cell barrier.

The schematic description of the evolution of the gastroresistant microparticles with varied $\mathrm{pH}$ is represented in Figure 3b. Figures 3c-d show SEM images of the Eudragit-based microcapsules loaded with rifampicin-PLGA nanoparticles and Figure 3e shows an empty Eudragit-based microcapsule where no surface roughness is observed due to the lack of inner PLGA nanoparticles. After incubation during $2 \mathrm{~h}$ at $37{ }^{\circ} \mathrm{C}$ in simulated gastric fluid $(0.1 \mathrm{~N} \mathrm{HCl}, \mathrm{pH}=1.03), \mathrm{the}$ morphology of the methacrylic acid-ethyl acrylate microparticles remained unaltered (Figure $2 \mathrm{c}$ and $3 \mathrm{~h}$ ). Depending on the methacrylic copolymer formulation, the carboxyl/ester ratio can be adjusted to control the polymer dissolution $\mathrm{pH}$.

The used gastroresistant polymer dissolves at $\mathrm{pH}$ above 5.5. To corroborate it, we encapsulated rifampicin-loaded PLGA NPs (Figure 2d) within Eudragit ${ }^{\circledast}$ L 100-55 microcapsules and then we immersed them in a simulated intestinal fluid based on PBS ( $\mathrm{pH}$ 6.8) during $3 \mathrm{~h}$ at $37{ }^{\circ} \mathrm{C}$ observing an immediate release of the inner PLGA nanoparticles. To corroborate that the NPs observed were actually the initial PLGA NPs instead of a re-crystallization or new particles composed of the polymer (Eudragit), we labelled the inner PLGA NPs with gold NPs following our previously published protocol[25]. As can be observed in Figure $3 \mathrm{~h}$, the microparticles resisted the simulated gastric fluid but upon contact with the simulated intestinal fluid the inner PLGA NPs were released almost immediately (Figures $3 \mathrm{f}-\mathrm{g}$ ).To observe the degradation process we immersed the microparticles in the simulated intestinal fluid during a few seconds at a $\mathrm{pH}$ of 5.5 and we observed how partially degraded (Eudragit-based) microcapsules containing the interior PLGA NPs started to be released (Figures 3 $\mathrm{i}-\mathrm{j}$ ). After $3 \mathrm{~h}$ the shell made of Eudragit was totally dissolved and the inner PLGA NPs loaded with gold nanoparticles inside were released to the simulated intestinal fluid (PBS at pH 6.8 and at $37^{\circ} \mathrm{C}$ ) (Figure $3 \mathrm{k}$ ). Hence, we can conclude that the inner PLGA NPs were the ones we previously pre-loaded inside the microparticles.

a)

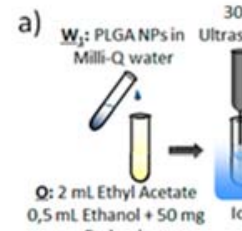

$$
\text { Eudragit }
$$
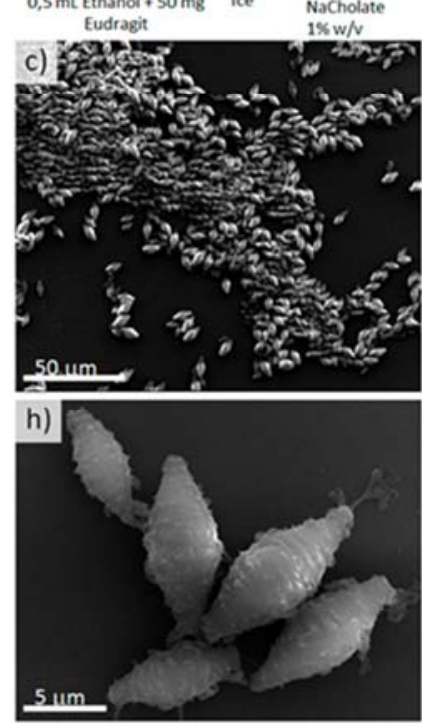

$30 \mathrm{~s}$,
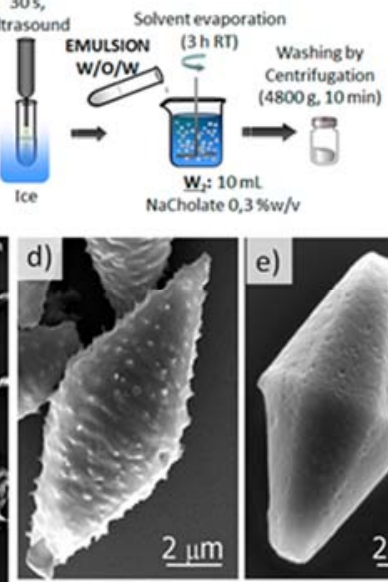

i)

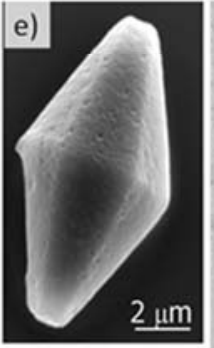

b)
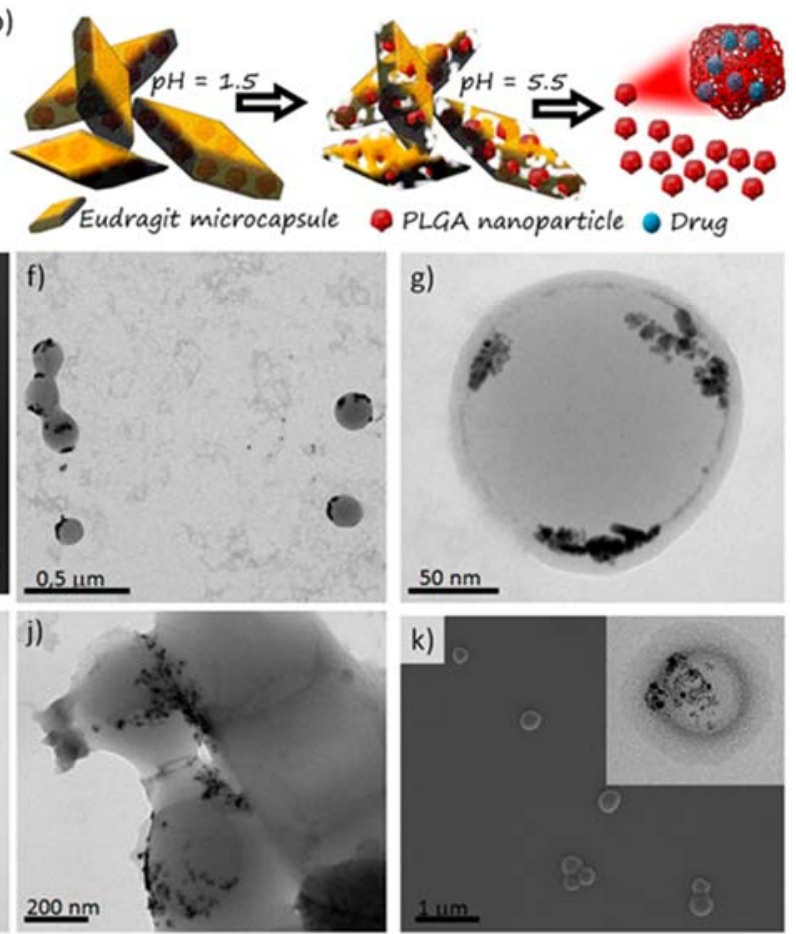

g)

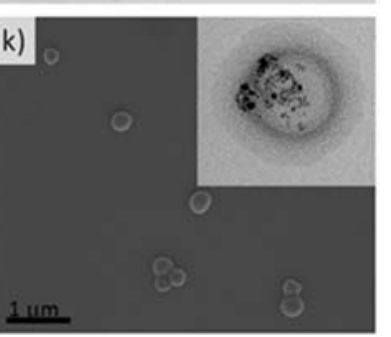

Figure 3. a) Schematic synthesis procedure for the synthesis of microparticles containing drug loaded-PLGA NPs by a double w/o/w emulsification and evaporation method. b) Schematic description of the evolution of the (Eudragit based) microparticles with $\mathrm{pH}$. c) SEM image of Eudragit microcapsules loaded with rifampicin-PLGA nanoparticles, d) SEM image, at a higher magnification, of a Eudragit microcapsule loaded with rifampicin-PLGA nanoparticles, e) SEM image of a non-loaded Eudragit microparticle, f) TEM image of Au loaded PLGA nanoparticles used as beacons to demonstrate the successful PLGA NPs encapsulation (Au nanoparticles appear as dark spots due to their high electronic density). g) TEM image of a zoomed Au loaded PLGA nanoparticle; Au NPs are trapped in the PLGA matrix. h) SEM image of Eudragit microcapsules loaded with Au-PLGA nanoparticles after $2 \mathrm{~h}$ in $\mathrm{HCl} 0.1 \mathrm{~N}$ at 37 ․ . i) TEM image of the Eudragit 
microcapsules loaded with Au-PLGA nanoparticles at $\mathrm{pH}=5.5$. The microcapsule is partially degraded and Au-PLGA NPs can be observed in the matrix. j) TEM image with a high magnification of microcapsules loaded with Au-PLGA nanoparticles at $\mathrm{pH}=5.5$. $\mathrm{k}$ ) SEM image of Au-PLGA nanoparticles after the microcapsule dissolution at $\mathrm{pH}=6.8$ after $3 \mathrm{~h}$ at 37 ㅇ. Inset, TEM image of one of the inner Au-PLGA NPs.

\section{Antimicrobial activity evaluation}

The resazurin assay was used to evaluate the MIC of nanoparticles and drugs against actively growing cultures of $M$. tuberculosis. Unloaded PLGA NPs, rifampicin-loaded PLGA NPs and free rifampicin were evaluated. The evaluated MIC of rifampicin was $0.015 \mu \mathrm{g} / \mathrm{mL}$, value in agreement with those described for strains susceptible to rifampicin[26]. The MIC of the empty PLGA NPs was $7.8 \mu \mathrm{g} / \mathrm{mL}$, hence indicating a moderate antituberculosis effect for the polymer PLGA.This intrinsic antituberculosis activity was attributed to the potential accelerated hydrolysis of the ester bonds of the PLGA, hence producing lactic and glycolic acids that would further acidify the media being this process autocatalytic[27]. As a consequence, increases in media acidification would in turn result in an enhanced bactericidal action for M. tuberculosis.

Interestingly, the MIC of the rifampicin-loaded PLGA NPs was $1.95 \mu \mathrm{g} / \mathrm{mL}$, which corresponded to $0.02 \mu \mathrm{g} / \mathrm{mL}$ of rifampicin (considering the $\mathrm{DL}$ ), which is a value very close to the one obtained for the free rifampicin $(0.015 \mu \mathrm{g} / \mathrm{mL})$. These experiments suggested that rifampicin was being released from the nanoparticles, so we designed an experiment to monitor kinetics of drug release. Nanoparticles were incubated in culture media at $\mathrm{pH} 7.0$ and the antituberculosis activity of nanoparticle-free supernatants was assayed against $M$. tuberculosis. We observed that in these conditions, free rifampicin was degraded upon time, rapidly after the first week, so we took this into consideration when analyzing the following results. After three days, the supernatants contained a $72.6 \%$ of the initial amount of rifampicin, and this value was $48.4 \%$ after 7 days (lower than the one obtained after three days due to rifampicin degradation). Longer incubation times resulted in much lower amounts of rifampicin, being $24.2 \%$ after 14 days and $6.0 \%$ after 25 days.

Sustained release was also observed for 7 days in blood serum by Booysen et al.[28] when using rifampicin-loaded PLGA-PEG NPs administered orally in a rat model whereas only during the first 2 days those authors were able to detect the antibiotic in blood serum when administered as a free (non-encapsulated) drug. In addition, Pandey and Khuller[29] also demonstrated the presence of systemic rifampicin 5 days after its oral administration in mice when encapsulated within PLGA NPs ( 200-300 nm) whereas the free drug was present only during the first $12 \mathrm{~h}$. We also observed that the rifampicin released from NPs incubated at $37^{\circ} \mathrm{C}$ during 25 days was still active and killed M. tuberculosis.

Under physiological conditions, mycobacteria reside in an acidic cellular compartment, so we investigated next the amount of rifampicin that would be released from the nanoparticles in such acidic conditions. For this, nanoparticles were incubated at $\mathrm{pH} 4.5$ and the antituberculosis activity of supernatants at different time points was determined by the resazurin method; results are given in Table 1. A considerable amount of rifampicin could be detected in the nanoparticle supernatants (40.3\%) after three days, indicating that the antibiotic was readily being released from the nanoparticles. We observed that the amount of rifampicin detected in the supernatants decreased after longer incubation times, which may be attributed to degradation of rifampicin in the culture media due to spontaneous reactions (as discussed above) [15]. In fact, after two weeks, $<6.0 \%$ of rifampicin could be detected in the supernatants at $\mathrm{pH} 4.5$, indicating that degradation of the antibiotic was faster under acidic conditions. In order to assess the latter, we performed a control assay from a solution of free rifampicin at pH 4.5 and detected that in fact, the amount of rifampicin that can be detected also decreases with time, although to a lesser extent than that obtained from the nanoparticle supernatants probably caused by a superior acidification of the medium due to the presence of lactic and glycolic acids as subproducts of the PLGA degradation (Table 1). Control experiments performed with the supernatants of empty nanoparticles did not show any antituberculosis activity even at the highest concentrations tested.

We tested next the impact of empty and antibiotic-loaded PLGA nanoparticles in the growth of $M$. tuberculosis. We first detected that empty nanoparticles produced a slight delay in growth of the $M$. tuberculosis culture, which is consistent with moderate intrinsic antituberculosis properties of PLGA detected in both the MIC assays and the murine alveolar macrophages infection model, as reported in this work and elsewhere in the literature[27]. Interestingly, we observed that rifampicin-loaded nanoparticles containing subinhibitory concentrations $(0.25 \times \mathrm{MIC})$ of rifampicin caused a further slight delay in the growth of M. tuberculosis in comparison with the same amount of empty NPs. Figure 4 shows the outcome of a representative experiment. This delay could be detected in terms of both $\mathrm{OD}_{600}$ (Figure $4 \mathrm{a}$ ) and also in the number of viable cells (Figure $4 b$ ). We attributed this effect to the release of the antibiotic from the nanoparticles. Given that rifampicin was used at a subinhibitory concentration, it only delayed but not arrested bacterial growth.

Table 1. Bioassay-based quantification of rifampicin released from PLGA NPs, in comparison with the amount detected from a free solution of rifampicin.

\begin{tabular}{|c|c|c|c|c|}
\hline $\mathrm{pH}$ & Days & \% Rif detected from Rif-loaded NPs & \% Activity of a control Rif solution \\
\hline
\end{tabular}




\begin{tabular}{|c|c|c|c|}
\hline \multirow{4}{*}{4.5} & 3 & 40.3 & 98.1 \\
\cline { 2 - 4 } & 7 & 10.1 & 39.9 \\
\cline { 2 - 4 } & 14 & $<6.0$ & 6.0 \\
\cline { 2 - 4 } & 25 & $<6.0$ & $<6.0$ \\
\hline
\end{tabular}

In order to estimate the antimicrobial activity of the rifampicin-loaded PLGA nanoparticles in conditions similar to those encountered during antituberculosis treatment, we performed time-kill kinetics experiments at $\mathrm{pH} 4.5$. The $\mathrm{pH}$ of the early endosome in which $M$. tuberculosis resides, ranges from pH 6.2 to 4.5 depending on the macrophage activation state[30]. We found that the free and the encapsulated rifampicin had similar levels of bacterial eradication, both causing a reduction larger than 8-log in the number of viable bacteria after 14 days of incubation (Figure 5). Empty PLGA NPs showed partial antimicrobial activity (2.5 log reduction compared to the non-treated bacterial cells, after 21 days of incubation; Figure 5), which is consistent with the fact that the concentrations of empty NPs used in this experiment are below, but rather close to, the inhibitory concentrations of PLGA.

We investigated the impact of the treatment with free rifampicin or rifampicin-containing nanoparticles in the morphology of $M$. tuberculosis under both acidic and neutral conditions. As can be seen in Figure 6 , at any condition tested the roughness of the surface of the bacteria wall under acidic conditions increased compared to the cells treated at physiological $\mathrm{pH}$. Treatment with rifampicin either free or encapsulated enhanced the alterations observed in the cell shape and roughness.
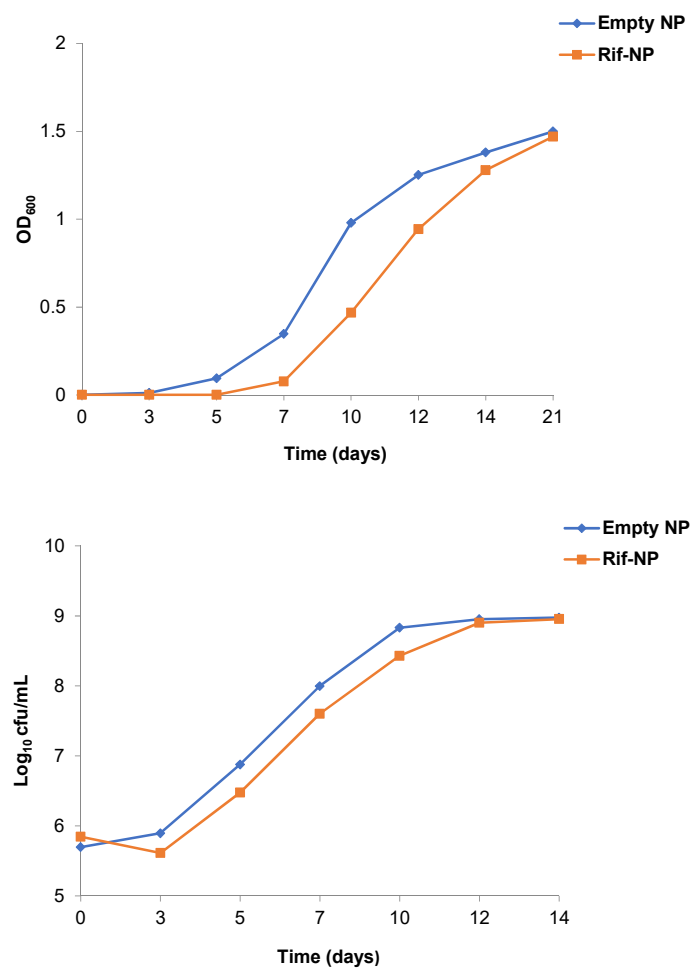

Figure 4.Impact of empty and rifampicin-loaded nanoparticles on the growth of $M$. tuberculosis on Middlebrook 7 H9 broth at $\mathrm{pH}$ 7.0. The rifampicin-loaded nanoparticles (red lines) contained a sub-inhibitory concentration of rifampicin and caused a slight delay in the growth of $M$. tuberculosis in comparison with the same amount of empty nanoparticles (black line) that was detected in terms of both OD600 (upper panel) and also in the number of viable cells (bottom panel), as shown in this representative experiment.

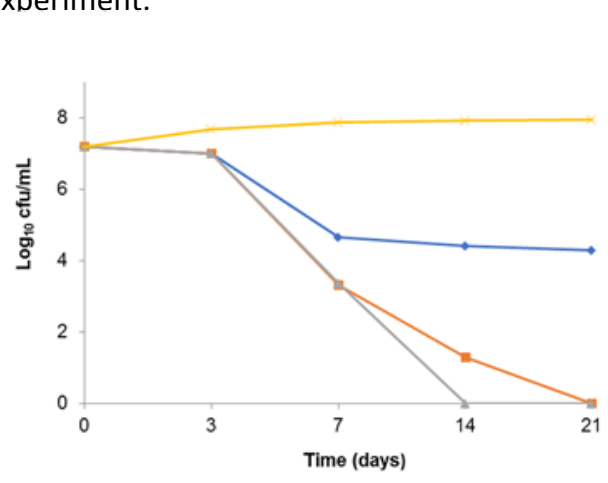


Figure 5.Time-kill kinetics at $\mathrm{pH}$ 4.5. High bacterial density $(1.5 \times 107 \mathrm{CFU} / \mathrm{ml})$ cultures of $M$. tuberculosis were incubated at $\mathrm{pH} 4.5$, conditions in which cells barely replicated (black line), and efficiency of several treatments for reducing the number of viable bacteria over time were compared. Treatment with $0.06-0.07 \mu \mathrm{g} / \mathrm{mL}$ of encapsulated rifampicin (between 4.5 and 6 times above the MIC) (red line) was as efficient as the free antibiotic at the same concentration (green line) to kill cells in a population, being capable of sterilizing the culture after two weeks. Intrinsic antimicrobial activity of PLGA was also detected (blue line), which was more than four orders of magnitude lower than that obtained for the antibiotic containing samples.

We also tested whether the antituberculosis activity of the rifampicin-loaded PLGA nanoparticles was retained after being released from the Eudragit microparticles. For this, the complete Matryoshka MPs were immersed in PBS at pH=7.4 in order to release the nanoparticles that were tested for antituberculosis activity in vitro. Nanoparticles containing rifampicin were effective at doses above $0.035 \mathrm{mg} / \mathrm{mL}$ whereas empty (without antibiotic) PLGA nanoparticles released from MPs did not show any antituberculosis effect at the highest dose tested $(1.12 \mathrm{mg} / \mathrm{mL})$.

\section{pH}

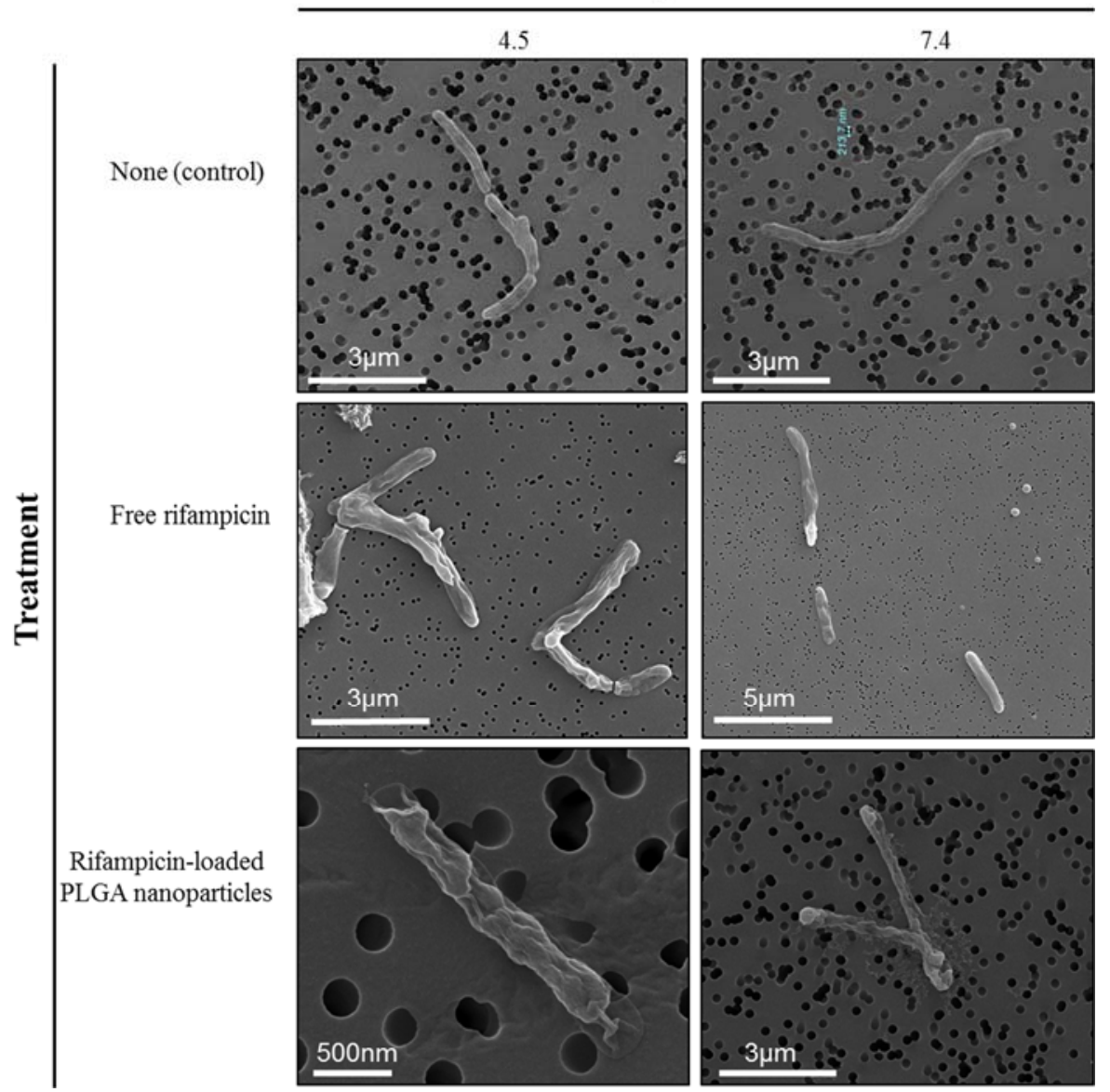

Figure 6.SEM photographs of the $M$. tuberculosis bacteria in contact with free rifampicin or with rifampicin-loaded nanoparticles under acidic $(\mathrm{pH} 4.5)$ and neutral $(\mathrm{pH} 7.4)$ conditions.

\section{Cell viability assay}

To study the influence of different PLGA formulations on cell viability, the potential cytotoxic effect of unloaded-PLGA nanoparticles, rifampicin-loaded PLGA nanoparticles and free rifampicin were assayed in two different cell lines, TC7 $\mathrm{Caco} 2$ and $\mathrm{MH}-\mathrm{S}$ murine alveolar macrophages. Caco2 cell line was selected because it is one of the most used in vitro models to study the intestinal transport when drugs are orally administrated. The results depicted in Figure S3 showed that both, rifampicin-loaded PLGA nanoparticles and free rifampicin did not induce cytotoxicity in the range of concentrations tested (0.0625-1 mg/mL PLGA nanoparticles and 0.5-8 $\mu \mathrm{g} / \mathrm{mL}$ of rifampicin).

MH-S murine alveolar macrophages were chosen because infected macrophages are the target where the drug has to exert its action. We tested unloaded and rifampicin loaded PLGA NPs with rifampicin as well as free rifampicin at serum concentrations $(4 \mu \mathrm{g} / \mathrm{mL}$ and $24 \mu \mathrm{g} / \mathrm{mL})$, during $72 \mathrm{~h}$. The results showed that the PLGA NPs empty or with rifampicin 
were not toxic for the macrophages at plasma concentrations. Moreover, it was not found any significant effect when free rifampicin was added to the macrophages (Figure S4).

\section{Evaluation of $\mathrm{CacO} 2$ cell monolayer integrity and visualization of epithelial monolayers by transmission electron} microscopy (TEM)

As we mentioned in the experimental section, the integrity of Caco2 monolayers on membrane supports was assessed by TEER measurements and LY rejection assays. TEER values steadily increased during Caco2 cell differentiation, reaching a plateau value around 28 days after seeding, tight junctions were formed after that time and the monolayer integrity increased over time (data not shown). The medium TEER value achieved was $500 \Omega \mathrm{cm}^{2}$. In general, a wide range of TEER values have been reported in the literature for these Caco2 cell clones. It has also been described that this heterogeneity in TEER measurements can be due to diverse factors such as cell passage number, medium composition and cell culture period.

At that moment, the LY permeability, a fluorescent indicator of cell monolayer integrity, was evaluated in the apical to basolateral direction across Caco2 monolayers. In general, no fluorescence intensity was detected in the basal compartment, indicating the formation of intact epithelial monolayers with well-formed tight junctions. As selection criteria, only monolayers with \% LY rejection values $\geq 99.9$ were selected to assess the integrity of the monolayers within the course of the experiment. Immediately after, a second value of TEER was determined to control the status and the integrity of the cellular monolayer at the end of the permeability experiment. Along with the LY rejection values mentioned above, changes lower than $5 \%$ in TEER values compared with the initial readings were taken as an indicator of the maintenance of monolayer integrity.

Finally, the precise formation of a homogenous Caco2 epithelial barrier (Figure 2e) was monitored using a slightly modified TEM protocol, previously reported [31]. In addition, TEM imaging allowed us to visualize the morphological organization of the epithelial barrier in detail. Thus, after four weeks in culture, TC7 Caco2 cells spontaneously formed a simple uniform epithelial layer with a structural organization similar to that of differentiated intestinal epithelial cells on the apical side of the transwell filters (Figure 7a3). This monolayer was constituted by polarized cuboidal-shaped cells that present a continuous brush border at their apical surface. The prominent brush border was composed of well-ordered and dense microvilli, presenting great homogeneity with regard to dimensions within and between cells. Different organelles such as mitochondria, lysosomes, and the nucleus were observed in the intracellular compartments (Figure S5). It is important to mention that tight junctions, the major subcellular structures in paracellular transport route, were visualized as intercellular contact areas between the membranes of two neighboring epithelial cells (Figure S5b-c). Other types of cell-cell junctions, such as adherens junctions and desmosome were also visualized (Figure S5b-c). Typical cell membrane folding, called interdigitations, were encountered between cells in the artificial epithelial monolayer (Figure $7 \mathrm{a} 3$ and Figure S5d). Altogether, these results confirmed that artificial Caco2 cell monolayers closely mimic the characteristics of the in vivo barrier and, therefore, maintained the adequate morphological organization and integrity to carry out nanoparticle transport experiments.

\section{Permeability assay through the artificial membrane assay}

The in vitro intestinal transport of rifampicin-loaded PLGA-based nanoparticles was studied in TC7 Caco2 cells after the incubation with growing concentrations of nanoparticle suspensions for $4 \mathrm{~h}$. TEM visualization of basolateral side samples (Figure 7a2) showed that, in all cases studied, a significant amount of rifampicin-loaded PLGA nanoparticles permeated through the artificial epithelial monolayer.

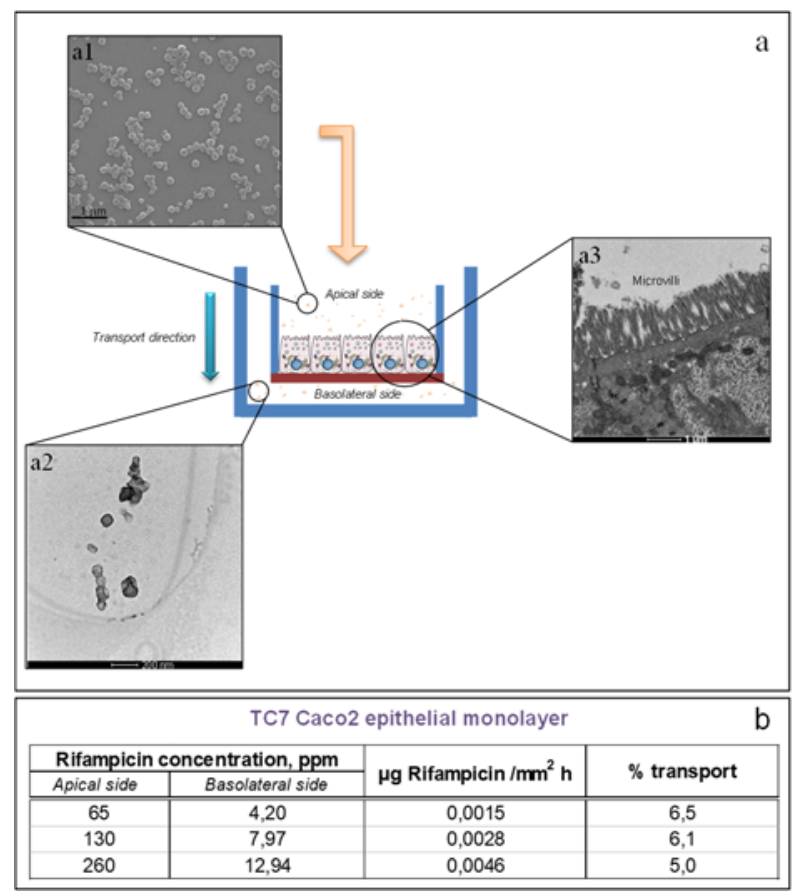


Figure 7. a) Permeability assay through the artificial membrane assay. SEM image of rifampicin loaded PLGA NPS before transport assay (a1); TEM image of rifampicin loaded PLGA NPs after transport assay (a2) and TEM image of morphology of TC7 Caco2 epithelial cell barrier (a3). b) Rifampicin concentration (ppm) presented in apical and basolateral side in TC7 Caco2 epithelial barrier. Rifampicin flux and transport data are also shown in both epithelial monolayers.

In addition, to complete the permeability study we quantified the transported rifampicin concentration presented in basolateral side samples by HPLC analysis. As shown in Figure 7b, the amount of transported drug that passed through Caco2 monolayers increased in a concentration-dependent manner, representing approximately $6 \%$ of the initial drug amount added to the apical chamber. These values further confirmed the results obtained by TEM imaging, indicating that the flux of rifampicin-loaded PLGA nanoparticles through TC7 Caco2 monolayer was $0.0046 \mu \mathrm{g} / \mathrm{mm}^{2} \mathrm{~h}$ (Figure $7 \mathrm{~b}$ ). It is important to mention that we cannot discard that a part of this rifampicin could be free drug that has been released from the loaded PLGA NPs before or/and after their translocation through the artificial epithelial membrane.

On the other hand, the effect of NP suspensions on the monolayer integrity was investigated by monitoring TEER values after permeability experiments. Our data indicated that the modification of post-experiment TEER values was lower than $5 \%$ in relation to the initial TEER values (data not shown), suggesting that the opening of tight junctions did not happen in the cell monolayer after nanoparticle suspensions exposure.

Taken all together, these results suggest that epithelial cells were able to uptake rifampicin-loaded PLGA NPs and those were able to translocate to the basolateral side. This means that in a potential in vivo application the nanoparticles would be able to likely reach systemic circulation. We used ester-terminated PLGA NPs to avoid electrical charge and hinder mucus adsorption. On the other hand, antibiotic NPs with reduced sizes (145 nm) were synthesized to favour the potential transcytosis by the Microfold cells ( $M$ cells) present in the gut-associated lymphoid tissue of the Peyer's patches in a hypothetical in vivo application.

\section{Antimycobacterial effect of rifampicin-loaded PLGA nanoparticles in murine alveolar macrophages infection model}

Finally, we investigated the proliferation of $M$. tuberculosis inside macrophages under the treatment with unloadedPLGA NPs, rifampicin-loaded PLGA NPs and free rifampicin at serum concentrations ( 4 and $24 \mu \mathrm{g} / \mathrm{mL}$ ). We found that at those two concentrations the exposure to PLGA NPs loaded with rifampicin caused a decrease in the growth of $M$. tuberculosis of 3.3 and 3.6 times respectively, compared with free rifampicin until day 4 (Figure $8 a-b$ ). It is possible that the effect can be stronger with a longer exposition, as it was observed when the bacteria was exposed to rifampicinloaded PLGA NPs (Figure 5) and also as it was reported by others in experiments with BCG in murine macrophages[8,32].

The effect observed after the treatment with rifampicin-loaded PLGA NPs at maximum concentration $(24 \mu \mathrm{g} / \mathrm{mL})$ could be related with the synergy observed between the drug and PLGA, since the final concentration of PLGA added was
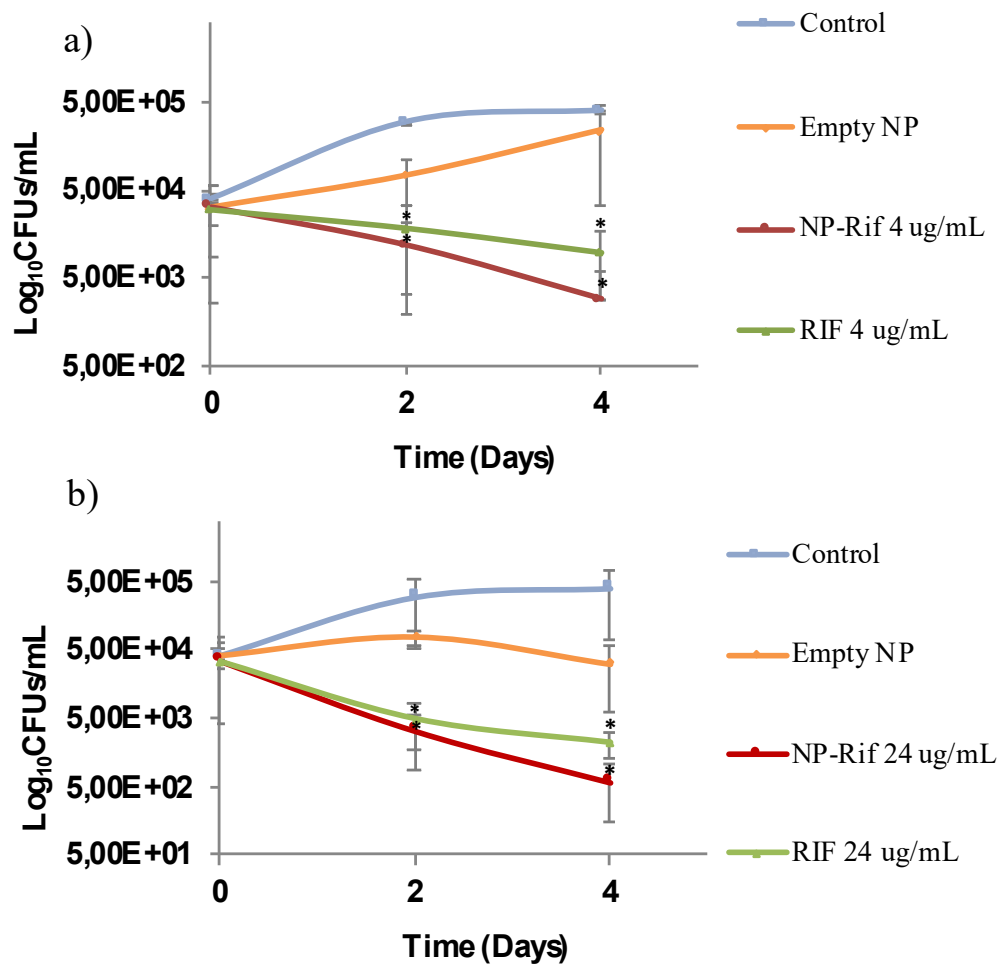
Figure 8. Growth of H37Rv strain inside MH-S macrophages after the treatment with empty PLGA NPs, rifampicin-loaded PLGA NPS and free rifampicin. (A) Minimum serum concentration $(4 \mu \mathrm{g} / \mathrm{mL})$. (B) Maximum serum concentration $(24 \mu \mathrm{g} / \mathrm{mL})$. Data are expressed as the average and standard deviation of triplicates of at least three independent experiments. ${ }^{*} \mathrm{P}<0,05$ (Compared with $\mathrm{MH}-\mathrm{S}$ control that was infected with H37Rv without NPs treatment, Mann-Whitney U test, GraphPad Software Inc.)

higher $(23.9 \mu \mathrm{g} / \mathrm{mL})$ than the MIC value $(7.8 \mu \mathrm{g} / \mathrm{mL})$. However, in the minimum concentration $(4 \mu \mathrm{g} / \mathrm{mL})$, the effect observed can be related exclusively to the drug since the PLGA concentration present was lower $(3.9 \mu \mathrm{g} / \mathrm{mL})$ than the $\mathrm{MIC}$ value.

On the other hand, we also found that empty PLGA NPs had some antimicrobial activity when the same quantity of PLGA as the one contained in rifampicin-loaded PLGA NPs, was added (Figure 8a-b). Again, the concentration was higher $(51.5-309 \mu \mathrm{g} / \mathrm{mL})$ than the MIC value. The same tendency was observed in the time-kill kinetics experiments and also by Lawlor et al. [26] in THP-1 macrophages using PLGA microparticles, who suggested that the antimicrobial effect could be related with the size and morphology of the particles used. It is important to mention that the approach here reported could also be used to encapsulate the second line injectable tuberculosis drugs utilized for the treatment of multidrugresistant tuberculosis that generally present poor efficacy, are more toxic and require prolonged treatments[33].

\section{Discussion}

The targeted drug delivery to the intracellular compartments of infected alveolar macrophages where $M$. tuberculosis resides and multiplies could be a promising approach to improve the antibiotics therapeutic efficacy in the treatment of this disease. In passive targeting, organic and inorganic NPs are rapidly internalized within cells to be degraded as part of the innate immune response against any foreign body. Together with granulocytes, resident or circulating macrophages as well as dendritic cells are all specialized in this clearance. Independently of the route of cellular entry (i.e., phagocytosis, macropinocytosis, clathrin or caveolinmediated endocytosis, etc.), the internalized NPs remain inside intracellular vesicles where enzymatic and acidic degradation take place. This NP uptake depends on the macrophage population considered and on its activation state[34].As we mentioned before, Kalluru et al. [8] demonstrated that whereas rifampicin-loaded PLGA NPs are internalized within acidic phagolysosomes, Mycobacterium bovis BCG remained in early phagosomes using BCG-infected mouse bone macrophages and also RAW mouse macrophages. However, even though co-localization within the same vesicle was not achieved, drug diffusion into the cytosol cleared the infection. We postulated that intracellular accumulation and slow antibiotic release can be achieved using orally administered antibiotic-loaded PLGA NPs if they were able to cross the intestinal epithelium into the blood stream with the sufficient blood circulation half-life to reach $M$. tuberculosis-infected alveolar macrophages. Thus, the encapsulation of antituberculosis drugs in nanoparticle delivery systems could also increase the drug bioavailability and, therefore, reduce drug dosage frequency. This fact would also allow decreasing treatment-related side effects (i.e., hepatotoxicity, skin reactions, gastrointestinal disturbances and immunological reactions) and improve drug regime compliance by the patient, two of the main clinical problems in the current treatment of tuberculosis. Other factor that can contribute to improve the patient adherence to the treatment is the way in which therapy is administered. In that sense, oral drug administration is the preferable route for long-term therapy due to its noninvasive character, simplicity and patient compliance. However, the main disadvantage of this administration route is the variability in the drug absorption due to degradation by the stomach acidic $\mathrm{pH}$ and lytic enzymes. Our study demonstrates that free rifampicin degrades over time under culture conditions (Table 1) and even more under acidic conditions in agreement with the previous literature [15].To overcome this drawback, rifampicin loaded NPs were encapsulated inside MPs based on methacrylic acid-ethyl acrylate copolymer, which remains insoluble in gastric conditions and dissolves in the intestinal fluid. In our case, we selected a polymer based on an anionic methacrylic acid-ethyl acrylate copolymer to release its cargo (antibiotic-loaded nanoparticles) at the duodenum pH taking advantage of the thin mucus layer of this first section of the small intestine and its high vascularization [35].In addition, at that level, it would be the nanoparticle carrier system that would act as a 
protector to minimize the rifampicin degradation induced by physiological conditions. Coatings targeting the intestine (based on hydroxypropyl methylcellulose phthalate) containing drug-loaded NPs have been used in vitro e in vivo in the systemic administration of insulin [17]. Those authors used a mixture of a non-acid dependent mucoadhesive polymer (Eudragit RS) with PLGA for the formulation of the inner insulin-loaded NPs and they demonstrated a prolonged hypoglycemic effect in a diabetic rat model after oral administration.

Previous to the permeability study, we provide evidence of no cytotoxicity of developed PLGA NPS on TC7 Caco2 and MH-S murine alveolar macrophages. These results are in agreement with previous reports conducted with PLGA NPs. Thus, Tukulula et al. [36] reported no cytotoxic effect of PLGA NPs and curdlan-conjugated PLGA NPs in THP1 macrophages over a $72 \mathrm{~h}$ period (dose range from 0.13 to $200 \mu \mathrm{g} / \mathrm{mL}$ ). In primary murine macrophages, thioridazine and rifampicin loaded-PLGA NPs and unloaded PLGA NPs did not significantly reduce cell viability throughout a 9 days treatment [37]. Also, PLGA NPs were not toxic to Caco2 cells after $24 \mathrm{~h}$ exposition, as long as the concentration was lower than $500 \mu \mathrm{g} / \mathrm{mL}$ [38]. An in vitro study carried out by Zhang et al. [39] to evaluate the cytotoxicity of PLGA NPs, demonstrated that they were well tolerated by Caco2 cells at concentrations below 25 $\mathrm{mg} / \mathrm{mL}$.

We used ester-terminated PLGA NPs to avoid electrical charge to hinder mucus adsorption and we synthesized antibiotic NPs with reduced sizes $(145 \mathrm{~nm})$ to favor the potential transcytosis by the Microfold cells ( $M$ cells) present in the gut-associated lymphoid tissue of the Peyer's patches in a hypothetical in vivo application. Bhavsar and Amiji [40] demonstrated that a high number of intestinal cells expressing the gene for green fluorescent protein (GFP) were observed when $200 \mathrm{~nm}$ gelatin NPs containing a plasmid encoding the GFP and in turn encapsulated within capsules were administered orally in rats which is indicative of a successful NP intracellular internalization.

The reduced size of the rifampicin-loaded PLGA NPs $(145 \mathrm{~nm})$ would favor the permeation through the intestinal epithelium reaching the systemic circulation. In this regard, Desai et al. demonstrated that $100 \mathrm{~nm}$ PLGA NPs orally administered in mice were able to diffuse through the submucosa layers whereas larger nano- and microparticles $(>500 \mathrm{~nm})$ remain trapped in the epithelial lining of the tissue [41]. Also, reduced NP sizes favor nanoparticle cellular internalization to achieve transcytosis considering that the paracellular transport constitutes only a $1 \%$ of the total mucosa surface area [42].Our in vitro permeability assay revealed that epithelial cells were able to uptake rifampicin-loaded PLGA NPs. Given that the paracellular transport is not viable due to the small size of interstitial space and that the intercellular junctions remain still tight during the permeability assay, the NPs internalization inside the cells may happen via endocytic uptake mechanisms. In that sense, previous works demonstrated that the uptake of PLGA NPs was reduced at $4{ }^{\circ} \mathrm{C}$ in $\mathrm{Caco} 2$ cells, indicating an endocytic uptake mechanism was involved in the NPs uptake for these cells [34]. The specific endocytosis pathways by which the particles may be translocated through the cells remain to be elucidated.

Also, we observed a sustained rifampicin release up to 25 days after incubation in culture media. Sustained release was also observed for 7 days in blood serum by Booysen et al. [28] when using rifampicin-loaded PLGA-PEG NPs administered orally in a rat model whereas only during the first 2 days those authors were able to detect the antibiotic in blood serum when administered as a free (non-encapsulated) drug. In addition, Pandey and Khuller [29] also demonstrated the presence of systemic rifampicin 5 days after its oral administration in mice when encapsulated within PLGA NPs (200-300 nm) whereas the free drug was present only during the first $12 \mathrm{~h}$.We also observed that the rifampicin released from NPs incubated at 37 o $\mathrm{C}$ during 25 days was still active and killed M. tuberculosis. Also, empty PLGA NPs showed intrinsic antituberculosis activity that was attributed to the potential accelerated hydrolysis of the ester bonds of the PLGA, hence producing lactic and glycolic acids that would further acidify the media being this process autocatalytic[27]. As a consequence, increases in media acidification would in turn result in an enhanced bactericidal action for M. tuberculosis. 
Finally, the assays in $M$. tuberculosis infected alveolar murine macrophages showed that the treatment with rifampicin-loaded PLGA NPs had an antimicrobial effect at both tested concentrations until day 4, significantly decreasing the intracellular bacterial load. It is possible that the effect can be stronger with a longer exposition, as it was observed when the bacteria was exposed to rifampicin-loaded PLGA NPs (Figure 5) and also as it was reported by others in experiments with BCG in murine macrophages $[8,32]$. It is important to mention that the approach here reported could also be used to encapsulate the second line injectable tuberculosis drugs utilized for the treatment of multidrug-resistant tuberculosis that generally present poor efficacy, are more toxic and require prolonged treatments [33].

\section{Conclusions}

By using the oil-in-water (O/W) emulsion solvent evaporation method it is possible to prepare monodisperse antibiotic-loaded PLGA-based NPs with high encapsulation efficiencies. Those antibiotic-loaded PLGA-based NPs can also be encapsulated within (Eudragit-based) monodisperse microcapsules using the double W/O/W emulsification and evaporation method obtaining antibiotic-loaded PLGA NPs encased within gastroresistant microparticles based on methacrylic acid-ethyl acrylate copolymers. Under gastric simulated conditions we demonstrated that the external coating remained unaltered and that the inner antibiotic-loaded NPs could be rapidly released under intestinal simulated conditions. We also demonstrated that those inner antibiotic-loaded NPs were able to cross an in vitro model of a simulated intestinal barrier composed of $\mathrm{CacO} 2$ cell monolayers. Therefore, those nanoparticles would be protected from gastric degradation and potentially reach systemic circulation. The antituberculosis action of the encapsulated rifampicin within the NPs was similar to that observed for the free drug indicating that the synthesis protocols did not alter the drug activity. This action was observed to be $\mathrm{pH}$ dependent considering both intracellular and extracellular milieus. We also demonstrated that those antibiotic-loaded NPs were non-cytotoxic on human cell lines but they were able to restrict the proliferation of M. tuberculosis inside infected macrophages. This novel delivery system shows a great potential for the successful targeted treatment of tuberculosis thanks to its demonstrated long-term release and its high cytocompatibility.

\section{Future perspective}

The increase of antibiotic resistant bacteria is a rising concern worldwide. The use of nanotechnological approaches can improve current antimicrobial treatments by providing with a rational usage of antibiotics. Targeted therapies based on drug-loaded nanoparticles with demonstrated benefits over the administration of the free drug will direct future research lines. Future research directions of the study here reported will be directed towards the in vivo evaluation of the antituberculosis effect of those antibiotic-loaded gastroresistant nanoparticles compared to the administration of the free drug in mice infected with M. tuberculosis strain H37Rv.

\section{Summary Points}

- Matryoshka-type gastroresistant microparticles containing antibiotic-loaded PLGA nanoparticles against Mycobacterium tuberculosis were produced by a multi-stage emulsification-evaporation method.

- The inner antibiotic-loaded nanoparticles were released under simulated intestinal conditions whereas the coating protected them from degradation under simulated gastric conditions.

- The Matryoshka-type procedure of encapsulation did not hinder the antituberculosis action of the encapsulated antibiotic rifampicin.

- A sustained antibiotic release can be obtained when using the encapsulated nanoparticles as well as a more effective elimination of M. tuberculosis when applied against infected macrophages compared to the use of the free drug.

- The potential co-localization of the drug-loaded nanoparticles with the intracellular pathogen in the same intracellular vesicle represents a promising approach to improve the antibiotic therapeutic efficacy in the treatment of the disease.

- Antibiotic-loaded PLGA nanoparticles were able to cross an in vitro model of intestinal barrier and demonstrated their suitability as effective drug carriers in the oral treatment of tuberculosis.

\section{Figure legends}

Figure 1. a) Synthesis procedure for the preparation of drug loaded PLGA NPs by single o/w emulsification and evaporation process. b)-c) SEM images of rifampicin loaded PLGA NPs. 
Figure 2. a) Scheme of the envisaged fate of the $\mathrm{pH}$-responsive Matryoshka-like micro- and nanoparticles for an efficient and controlled intracellular delivery of antibiotics against intracellular alveolar infected macrophages. b) SEM of Eudragit microcapsules containing drug-loaded PLGA nanoparticles. c) SEM of Eudragit microcapsules after $2 \mathrm{~h}$ in $\mathrm{HCl} 0.1 \mathrm{~N}$ at 37 oC (simulated gastric conditions). d) SEM of the rifampicin-loaded PLGA nanoparticles loaded inside the external microcapsules. e) TEM image of morphology of TC7 Caco2 epithelial cell barrier.

Figure 3. a) Schematic synthesis procedure for the synthesis of microparticles containing drug loaded-PLGA NPs by a double w/o/w emulsification and evaporation method. b) Schematic description of the evolution of the (Eudragit based) microparticles with $\mathrm{pH}$. c) SEM image of Eudragit microcapsules loaded with rifampicin-PLGA nanoparticles, d) SEM image, at a higher magnification, of a Eudragit microcapsule loaded with rifampicin-PLGA nanoparticles, e) SEM image of a non-loaded Eudragit microparticle, f) TEM image of Au loaded PLGA nanoparticles used as beacons to demonstrate the successful PLGA NPs encapsulation (Au nanoparticles appear as dark spots due to their high electronic density). g) TEM image of a zoomed Au loaded PLGA nanoparticle; Au NPs are trapped in the PLGA matrix. h) SEM image of Eudragit microcapsules loaded with Au-PLGA nanoparticles after $2 \mathrm{~h}$ in $\mathrm{HCl} 0.1 \mathrm{~N}$ at 37 oC. i) TEM image of the Eudragit microcapsules loaded with Au-PLGA nanoparticles at $\mathrm{pH}=5.5$. The microcapsule is partially degraded and Au-PLGA NPs can be observed in the matrix. j) TEM image with a high magnification of microcapsules loaded with Au-PLGA nanoparticles at $\mathrm{pH}=5.5$. $\mathrm{k}$ ) SEM image of Au-PLGA nanoparticles after the microcapsule dissolution at $\mathrm{pH}=6.8$ after $3 \mathrm{~h}$ at 37 ㅇ. Inset, TEM image of one of the inner Au-PLGA NPs.

Figure 4. Impact of empty and rifampicin-loaded nanoparticles on the growth of $M$. tuberculosis on Middlebrook $7 \mathrm{H} 9$ broth at $\mathrm{pH}$ 7.0. The rifampicin-loaded nanoparticles (red lines) contained a sub-inhibitory concentration of rifampicin and caused a slight delay in the growth of $M$. tuberculosis in comparison with the same amount of empty nanoparticles (black line) that was detected in terms of both OD600 (upper panel) and also in the number of viable cells (bottom panel), as shown in this representative experiment.

Figure 5. Time-kill kinetics at $\mathrm{pH}$ 4.5. High bacterial density $(1.5 \times 107 \mathrm{CFU} / \mathrm{ml})$ cultures of $M$. tuberculosis were incubated at $\mathrm{pH} 4.5$, conditions in which cells barely replicated (black line), and efficiency of several treatments for reducing the number of viable bacteria over time were compared. Treatment with $0.06-0.07 \mu \mathrm{g} / \mathrm{mL}$ of encapsulated rifampicin (between 4.5 and 6 times above the MIC) (red line) was as efficient as the free antibiotic at the same concentration (green line) to kill cells in a population, being capable of sterilizing the culture after two weeks. Intrinsic antimicrobial activity of PLGA was also detected (blue line), which was more than four orders of magnitude lower than that obtained for the antibiotic containing samples

Figure 6. SEM photographs of the $M$. tuberculosis bacteria in contact with free rifampicin or with rifampicin-loaded nanoparticles under acidic ( $\mathrm{pH} 4.5)$ and neutral $(\mathrm{pH} 7.4)$ conditions.

Figure 7. a) Permeability assay through the artificial membrane assay. SEM image of rifampicin loaded PLGA NPS before transport assay (a1); TEM image of rifampicin loaded PLGA NPs after transport assay (a2) and TEM image of morphology of TC7 Caco2 epithelial cell barrier (a3). b) Rifampicin concentration (ppm) presented in apical and basolateral side in TC7 Caco2 epithelial barrier. Rifampicin flux and transport data are also shown in both epithelial monolayers

Figure 8. Growth of H37Rv strain inside MH-S macrophages after the treatment with empty PLGA NPs, rifampicin-loaded PLGA NPs and free rifampicin. (A) Minimum serum concentration $(4 \mu \mathrm{g} / \mathrm{mL})$. (B) Maximum serum concentration $(24 \mu \mathrm{g} / \mathrm{mL})$. Data are expressed as the average and standard deviation of triplicates of at least three independent experiments. ${ }^{*} \mathrm{P}<0,05$ (Compared with $\mathrm{MH}-\mathrm{S}$ control that was infected with H37Rv without NPs treatment, Mann-Whitney U test, GraphPad Software Inc).

\section{Table Legends}

Table 1. Bioassay-based quantification of rifampicin released from PLGA NPs, in comparison with the amount detected from a free solution of rifampicin.

\section{References:}

Supplementary data to this article can be found online at XXXX

1. Carryn S, Chanteux H, Seral C, Mingeot-Leclercq M-P, Van Bambeke F, Tulkens PM. Intracellular pharmacodynamics of 
antibiotics. Infect. Dis. Clin. North Am.. 17(3), 615-34 (2003). A

2. WHO | Global tuberculosis report 2017. WHO [Internet]. (2017). Available from:

http://www.who.int/tb/publications/global_report/en/.

3. Griffiths G, Nyström B, Sable SB, Khuller GK. Nanobead-based interventions for the treatment and prevention of tuberculosis. Nat. Rev. Microbiol. 8(11), 827-834 (2010).

4. Noorlander CW, Kooi MW, Oomen AG, Park MV, Vandebriel RJ, Geertsma RE. Horizon scan of nanomedicinal products. Nanomedicine. 10(10), 1599-1608 (2015).

5. Pissuwan D, Cortie $\mathrm{CH}$, Valenzuela SM, Cortie MB. Functionalised gold nanoparticles for controlling pathogenic bacteria. Trends Biotechnol. 28(4), 207-213 (2010).

6. Pornpattananangkul D, Zhang L, Olson S, et al. Bacterial Toxin-Triggered Drug Release from Gold Nanoparticle-Stabilized Liposomes for the Treatment of Bacterial Infection. J. Am. Chem. Soc. 133(11), 4132-4139 (2011).

7. Xiong M-H, Bao Y, Yang X-Z, Wang Y-C, Sun B, Wang J. Lipase-Sensitive Polymeric Triple-Layered Nanogel for "On-Demand" Drug Delivery. J. Am. Chem. Soc. 134(9), 4355-4362 (2012).

8. Kalluru R, Fenaroli F, Westmoreland D, et al. Poly(lactide-co-glycolide)-rifampicin nanoparticles efficiently clear Mycobacterium bovis BCG infection in macrophages and remain membrane-bound in phago-lysosomes. J. Cell Sci. 126(14), 3043-3054 (2013).

** This manuscript represents a clear example of how intracellular co-localization of the antibiotic and the pathogen renders a better antimicrobial action than the administration of the free drug

9. Patel PV, Kumar S, Vidya GD, Patel A, Holmes JC, Kumar V. Cytological assessment of healing palatal donor site wounds and grafted gingival wounds after application of ozonated oil: an eighteen-month randomized controlled clinical trial. Acta Cytol. 56(3), 277-84 (2012).

10. Suen W-LL, Chau Y. Size-dependent internalisation of folate-decorated nanoparticles via the pathways of clathrin and caveolae-mediated endocytosis in ARPE-19 cells. J. Pharm. Pharmacol. 66(4), 564-573 (2014).

11. Voigt J, Christensen J, Shastri VP. Differential uptake of nanoparticles by endothelial cells through polyelectrolytes with affinity for caveolae. Proc. Natl. Acad. Sci. U. S. A. 111(8), 2942-7 (2014).

12. Mitragotri S, Burke PA, Langer R. Overcoming the challenges in administering biopharmaceuticals: formulation and delivery strategies. Nat. Rev. Drug Discov. 13(9), 655-672 (2014).

13. Patel BK, Parikh RH, Aboti PS. Development of oral sustained release rifampicin loaded chitosan nanoparticles by design of experiment. J. Drug Deliv. 2013, 370938 (2013).

** High drug loadings up to $43 \mathrm{wt} . \%$ can be reached by using chitosan as an encapsulation matrix and following a rational nanoparticle design.

14. Campbell EA, Korzheva N, Mustaev A, et al. Structural mechanism for rifampicin inhibition of bacterial rna polymerase. Cell [Internet]. 104(6), 901-12 (2001). Available from: http://www.ncbi.nlm.nih.gov/pubmed/11290327.

15. Singh $\mathrm{H}$, Bhandari R, Kaur IP. Encapsulation of Rifampicin in a solid lipid nanoparticulate system to limit its degradation and interaction with Isoniazid at acidic pH. Int. J. Pharm. 446(1-2), 106-111 (2013).

* This manuscript describes that the gastroprotection of rifampicine can be enhanced by using nanoencapsulation. Also that it is neccesray to pay attention to the potential drug-drug intercations when coencapsulating multiple drugs.

16. Pridgen EM, Alexis F, Kuo TT, et al. Transepithelial Transport of Fc-Targeted Nanoparticles by the Neonatal Fc Receptor for Oral Delivery. Sci. Transl. Med. [Internet]. 5(213), 213ra167-213ra167 (2013).

17. Wu ZM, Zhou L, Guo XD, et al. HP55-coated capsule containing PLGA/RS nanoparticles for oral delivery of insulin. Int. J. Pharm. 425(1-2), 1-8 (2012).

18. Médigue C, Cole ST, Camus J-C, Pryor MJ. Re-annotation of the genome sequence of Mycobacterium tuberculosis H37Rv. Microbiology 148(10), 2967-2973 (2002).

19. Khajuria V, Tandon V, Arora E, Choudhary N, Gillani Z. Effect of Carum carvi, a herbal bioenhancer on pharmacokinetics of antitubercular drugs: A study in healthy human volunteers. Perspect. Clin. Res. 5(2), 80 (2014).

20. Fahimi F, Tabarsi P, Kobarfard F, et al. Isoniazid, rifampicin and pyrazinamide plasma concentrations 2 and $6 \mathrm{~h}$ post dose in patients with pulmonary tuberculosis. Int. J. Tuberc. Lung Dis. 17(12), 1602-1606 (2013).

21. Peloquin CA. Therapeutic drug monitoring in the treatment of tuberculosis. Drugs 62(15), 2169-83 (2002).

22. Babalik A, Babalik A, Mannix S, Francis D, Menzies D. Therapeutic drug monitoring in the treatment of active tuberculosis. Can. Respir. J. 18(4), 225-9 (2011).

23. Motta I, Calcagno A, Bonora S. Pharmacokinetics and pharmacogenetics of anti-tubercular drugs: a tool for treatment optimization? Expert Opin. Drug Metab. Toxicol. 14(1), 59-82 (2018).

24. Kashi TSJ, Eskandarion S, Esfandyari-Manesh M, et al. Improved drug loading and antibacterial activity of minocyclineloaded PLGA nanoparticles prepared by solid/oil/water ion pairing method. Int. J. Nanomedicine. 7, 221-34 (2012).

25. Luque-Michel E, Larrea A, Lahuerta C, et al. A simple approach to obtain hybrid Au-loaded polymeric nanoparticles with a tunable metal load. Nanoscale 8(12), 6495-6506 (2016).

26. Martin A, Camacho M, Portaels F, Palomino JC. Resazurin microtiter assay plate testing of Mycobacterium tuberculosis susceptibilities to second-line drugs: rapid, simple, and inexpensive method. Antimicrob. Agents Chemother. 47(11), 36169 (2003).

27. Lawlor C, O'Connor G, O'Leary S, et al. Treatment of Mycobacterium tuberculosis-Infected Macrophages with Poly(LacticCo-Glycolic Acid) Microparticles Drives NFkB and Autophagy Dependent Bacillary Killing. PLoS One 11(2), e0149167 (2016). 
28. Booysen LLIJ, Kalombo L, Brooks E, et al. In vivo/in vitro pharmacokinetic and pharmacodynamic study of spray-dried poly(dl-lactic-co-glycolic) acid nanoparticles encapsulating rifampicin and isoniazid. Int. J. Pharm. 444(1-2), 10-17 (2013).

29. Pandey R, Khuller GK. Oral nanoparticle-based antituberculosis drug delivery to the brain in an experimental model. J. Antimicrob. Chemother. 57(6), 1146-1152 (2006).

30. Vandal OH, Nathan CF, Ehrt S. Acid resistance in Mycobacterium tuberculosis. J. Bacteriol. 191(15), 4714-21 (2009).

31. Ye D, Dawson KA, Lynch I. A TEM protocol for quality assurance of in vitro cellular barrier models and its application to the assessment of nanoparticle transport mechanisms across barriers. Analyst 140(1), 83-97 (2015).

32. Hirota K, Hasegawa T, Nakajima T, et al. Delivery of rifampicin-PLGA microspheres into alveolar macrophages is promising for treatment of tuberculosis. J. Control. Release 142(3), 339-346 (2010).

33. Quenard F, Fournier PE, Drancourt M, Brouqui P. Role of second-line injectable antituberculosis drugs in the treatment of MDR/XDR tuberculosis. Int. J. Antimicrob. Agents. 50(2), 252-254 (2017).

34. Weissleder R, Nahrendorf M, Pittet MJ. Imaging macrophages with nanoparticles. Nat. Mater. 13(2), 125-138 (2014).

35. Ensign LM, Cone R, Hanes J. Oral drug delivery with polymeric nanoparticles: The gastrointestinal mucus barriers. Adv. Drug Deliv. Rev. 64(6), 557-570 (2012).

36. Tukulula M, Hayeshi R, Fonteh $\mathrm{P}$, et al. Curdlan-Conjugated PLGA Nanoparticles Possess Macrophage Stimulant Activity and Drug Delivery Capabilities. Pharm. Res. 32(8), 2713-26 (2015).

37. Vibe CB, Fenaroli F, Pires D, et al. Thioridazine in PLGA nanoparticles reduces toxicity and improves rifampicin therapy against mycobacterial infection in zebrafish. Nanotoxicology. 10(6), 680-688 (2016).

38. Katsikari A, Patronidou C, Kiparissides C, Arsenakis M. Uptake and cytotoxicity of poly(d,I-lactide-co-glycolide) nanoparticles in human colon adenocarcinoma cells. Mater. Sci. Eng. B 165(3), 160-164 (2009).

39. Zhang X, Sun M, Zheng A, Cao D, Bi Y, Sun J. Preparation and characterization of insulin-loaded bioadhesive PLGA nanoparticles for oral administration. Eur. J. Pharm. Sci. 45(5), 632-638 (2012).

40. Bhavsar MD, Amiji MM. Gastrointestinal distribution and in vivo gene transfection studies with nanoparticles-inmicrosphere oral system (NiMOS). J. Control. Release. 119(3), 339-348 (2007).

41. Desai MP, Labhasetwar V, Amidon GL, Levy RJ. Gastrointestinal uptake of biodegradable microparticles: effect of particle size. Pharm. Res. 13(12), 1838-45 (1996).

42. Plapied L, Duhem N, des Rieux A, Préat V. Fate of polymeric nanocarriers for oral drug delivery. Curr. Opin. Colloid Interface Sci. 16(3), 228-237 (2011). 